\title{
Bifurcations of Normally Hyperbolic Invariant Manifolds and Consequences for Reaction Dynamics
}

\author{
Frédéric A. L. Mauguière* \\ School of Mathematics \\ University of Bristol \\ Bristol BS8 1TW \\ United Kingdom \\ Peter Collins \\ School of Mathematics \\ University of Bristol \\ Bristol BS8 1TW \\ United Kingdom \\ Gregory S. Ezrd† \\ Department of Chemistry and Chemical Biology \\ Baker Laboratory \\ Cornell University \\ Ithaca, NY 14853 \\ USA \\ Stephen Wiggins \\ School of Mathematics \\ University of Bristol \\ Bristol BS8 1TW \\ United Kingdom
}

(Dated: March 22, 2022) 


\begin{abstract}
In this paper we study the breakdown of normal hyperbolicity and its consequences for reaction dynamics; in particular, the dividing surface, the flux through the dividing surface (DS), and the gap time distribution. Our approach is to study these questions using simple, two degree-of-freedom Hamiltonian models where calculations for the different geometrical and dynamical quantities can be carried out exactly. For our examples, we show that resonances within the normally hyperbolic invariant manifold may, or may not, lead to a 'loss of normal hyperbolicity'. Moreover, we show that the onset of such resonances results in a change in topology of the dividing surface, but does not affect our ability to define a DS. The flux through the DS varies continuously with energy, even as the energy is varied in such a way that normal hyperbolicity is lost. For our examples the gap time distributions exhibit singularities at energies corresponding to the existence of homoclinic orbits in the DS, but these singularities are not associated with loss of normal hyperbolicity.
\end{abstract}

PACS numbers: 05.45.-a, 45.10.Na, 82.20.Db, 82.20.-w 


\section{INTRODUCTION}

Transition state theory has played, and continues to play, a fundamental role in how we think of chemical reactions. There are many excellent reviews of the subject; see, for example, refs 1,2,3,4,5. The work of Wigner ${ }^{\sqrt{6}}$ highlighted the notion of the transition state as a dividing surface (DS) in phase space, having the property that trajectories crossed this surface (only once) in their evolution from reactants to products. This phase space point of view was relatively undeveloped for many years until the 1970's when such a dynamical theory was fully realised for two degrees of freedom (DoF) in the work of Pechukas, Pollak and Child $7 / 8 / 9|10| 11|12| 13$. In their work they realised the construction of such a DS having the 'no recrossing' property, and a central role was played by a saddle-like (in terms of stability) periodic orbit (PO) which serves as the 'anchor' for the definition of the DS. This PO was called a periodic orbit dividing surface (PODS). (This choice of name was perhaps unfortunate as the PO itself is not the DS [in phase space] but rather the boundary of the DS.) The work of Pechukas, Pollak and Child just cited was the first example of the use of a normally hyperbolic invariant manifold (NHIM) in the context of transition state theory (although see also ref. 14).

For more than two DoF a suitable generalisation of a saddle like (or 'hyperbolic') periodic orbit was required, and this was realised in the notion of a normally hyperbolic invariant manifold (NHIM). Explicit discussion of NHIMs in the context of reaction theory was in refs 15,16 (see also refs 17,18). However, the usefulness of NHIMs for the definition of phase space dividing surfaces was fully realised only after the development of methods for computing NHIMS and their associated DS. In particular, NHIMs could be computed using the classical Poincaré-Birkhoff normal form procedure, and its quantum analog 19220121 . However, the Poincaré-Birkhoff normal form procedure is local in nature since it involves a Taylor expansion about an appropriate saddle point. Practically, the expansion must be truncated at an appropriate order so that its ability to describe geometrical structures and dynamics at a desired accuracy must be assessed (and this is, typically, problem dependent). Very broadly speaking, the NHIM, and its associated local dynamics, are accurately described by the truncated normal form for energies 'close' to the energy of the saddle point. To date, our knowledge of NHIMs for specific problems relies heavily on the techniques used to compute them, in particular normal form theory. The local nature of these techniques makes 
NHIMs tools of limited utility in terms of our ability describe reaction dynamics at energies well above the reaction threshold. In particular, very little is known about how the NHIM behaves as we increase the energy above that of the saddle. We expect the normal form to 'break down' (in some not very precisely defined sense). However, the breakdown of the normal form and the breakdown of the NHIM are two separate issues. Questions concerning the existence and properties of the NHIM can in principle be considered independently of the methods used to compute the NHIM, when normal form approximations may no longer apply.

In this paper we study the behaviour of NHIMs as the energy above the saddle point is increased, and the dependence on energy of associated quantities related to reaction dynamics. Our strategy will be to avoid the use of normal forms by considering simple models that allow us to explicitly compute the NHIM, the DS, and the geometrical properties associated with reaction dynamics. We can view our approach as a decoupling of normal form issues from the dynamics of reaction. We then ask the question: "what happens to a NHIM as parameters (the energy being a very important parameter) are varied"? In other words, we study 'bifurcation of NHIMs'. But, more specifically for reaction dynamics, we want to understand how such bifurcation of NHIMs affects the various quantities (described below) used to describe reaction dynamics.

First, we need to describe some background regarding what we mean by the 'bifurcation of a NHIM' and then discuss our approach for studying these phenomena. Roughly speaking (for details, see Section II ) a normally hyperbolic invariant manifold has the property that the linearized growth rates normal to the manifold dominate the linearized growth rates tangent to the manifold. The nature of the dynamics within the invariant manifold can be arbitrary, provided the growth rate conditions are satisfied. Hence, there are several possibilities to consider:

- Bifurcation within the NHIM, but with the normal hyperbolicity conditions not affected.

- Breakdown of the normal hyperbolicity conditions.

- A combination of the above two phenomena - bifurcation within the NHIM and breakdown of the normal hyperbolicity conditions. 
How bifurcation within the NHIM occurs and how the normal hyperbolicity conditions can break down will be discussed in detail when we consider explicit models in Section III A. Briefly, there are two ways in which such bifurcations can occur. One is when a parameter in the Hamiltonian is varied, and the other is when the energy itself is varied. (We will be considering only Hamiltonian systems.) Of course, energy is a parameter, but its importance in understanding the dynamics is such that we highlight its effects explicitly.

Typically in bifurcation theory simple 'normal forms' are developed that embody the essential features of a particular bifurcation. We will follow a similar approach here. Our models will be two degree-of-freedom, separable, Hamiltonian systems where we have explicit control and understanding of the dynamics in such a way that we can model the three possibilities noted above. Modelling the three possibilities for separable, two DoF Hamiltonian systems is of course fairly straightforward, but that is the point. Our goal is to determine the effect that the three scenarios outlined above have on quantities that are used to describe reaction dynamics. In particular, we want to consider the effect on:

- The dividing surface (DS) separating reactants and products as a function of energy (the DS is defined and explained in Section III B).

- The flux through the DS as a function of energy.

- The gap time distribution as a function of energy (these quantities are defined and explained in Section IIB.

Despite the fact that our examples are very simple (completely integrable 2 DoF Hamiltonian systems), they allow for exact calculations and therefore provide benchmarks against which more complex examples can be compared. In particular, we note a previous study by $\mathrm{Li}$ and coworkers ${ }^{22}$ which suggests that the breakdown of normal hyperbolicity of the NHIM is responsible for the non definability of a relevant DS at high energy above the reaction threshold. This conclusion is contrary to that reached in the study of our model examples (see below). A study of Allahem and Bartsch ${ }^{23}$ asserts that the transition state loses, and then regains normal hyperbolicity as energy is varied. A study of Inarrea et a ${ }^{24}$ concludes that even though bifurcation of the NHIM can occur, dividing surfaces can still be defined. These three studies are concerned with Hamilltonian models that are much more complex than ours (although still 2 DoF). We also note an interesting study by Yang 25 , although the models studied there are not Hamiltonian. 
The organisation of this paper is as follow. We review the concepts of NHIMs in Section (II) where we discuss the definition of normal hyperbolicity in detail. In Section IIA we discuss the general form for the two DoF models that we study. These models allow us to explicitly compute NHIMs and quantify their geometrical and stability properties. In Section IIB we discuss concepts of phase space volumes, gap times, and reaction rates that are relevant for the present study. In Section IIIA we describe the two models for which we will carry out explicit calculations. In Section $\amalg I I B$ we discuss dividing surfaces and compute the flux through the dividing surfaces for our two examples, while Section IIIC deals with the computation of the gap time distribution. In Section IIID we discuss the loss of normal hyperbolicity and its consequences for the DS, flux, and gap time distribution in our examples. In Section [IV we present our conclusions. 


\section{NORMALLY HYPERBOLIC INVARIANT MANIFOLDS AND MODELS FOR THEIR BIFURCATION}

The notion of a normally hyperbolic invariant manifold (or NHIM) is by now a standard concept and tool in dynamical systems theory that is playing an increasingly important role in a variety of applications. The theoretical framework developed over the course of many years, beginning in the early part of the 20th century and reached a mature form in the works of Fenichel ${ }^{26 \mid 27 / 28}$ and Hirsch, Pugh, and Shub ${ }^{29}$. In ref. 30 a (relatively) elementary exposition of Fenichel's approach to NHIMs is given with some discussion of applications.

We begin by defining the notion of a normally hyperbolic invariant manifold (NHIM) in a continuous time setting (i.e. the setting of autonomous ordinary differential equations, rather than maps). There are numerous definitions that appear throughout the literature. We take the definition from ref. 31. From an 'applied' point of view the definition may appear somewhat abstract, leading to difficulties understanding how the definition can be applied in concrete settings. However, this level of abstraction actually provides a great deal of flexibility for applications, as we shall see when we consider explicit models for illustrating different types of bifurcation phenomena associated with NHIMs and reaction dynamics.

It is sufficient for our purpose to take phase space to be $\mathbb{R}^{2 n}$, which is a $C^{\infty}$ differentiable manifold. For inner products and norms on this space we will take the usual Euclidean structures. Phase space is taken to be even dimensional since we will be considering canonical Hamiltonian systems, i.e. we consider equations of the following form:

$$
\begin{aligned}
\dot{q}_{i} & =+\frac{\partial H}{\partial p_{i}}(q, p), \quad i=1, \ldots, n, \\
\dot{p}_{i} & =-\frac{\partial H}{\partial q_{i}}(q, p), \quad i=1, \ldots, n,
\end{aligned}
$$

for some function $H\left(q_{1}, \ldots, q_{n}, p_{1}, \ldots, p_{n}\right) \equiv H(q, p)$. We require the Hamiltonian $H(q, p)$ to be at least $C^{k+1}$ since we will assume the flow that it generates is at least $C^{k}$. In practice, this is not a restriction since the explicit Hamiltonians that we consider will be infinitely differentiable. However, in the theoretical description of NHIMs, it is important to explicitly specify the degree of differentiability of all geometrical objects for reasons that will become apparent as we develop the discussion of NHIMs further. Note that in this work we will have no need to consider $\mathbb{R}^{2 n}$ as a symplectic vector space, or to investigate the associated consequences of symplecticity; this is likely to be a very fruitful avenue for future 
research. The reason is the following. Roughly, in Hamiltonian dynamical systems stability properties are divided into 'elliptic' and 'hyperbolic', where bifurcation may occur when passing through the boundary between stability types. Results describing 'elliptic stability' (e.g. the Kolmogorov-Arnold-Moser (KAM) and Nekhoroshev theorems) rely heavily on the Hamiltonian structure for their proof. 'Hyperbolic stability' results, on the other hand, do not generally rely on the Hamiltonian structure, and that is true for the development of NHIMs. It would nevertheless be interesting to know what restrictions Hamiltonian structure places on the structure of NHIMs. For example, in ref. 32 it is proven that invariant tori in canonical, time independent, Hamiltonian systems cannot be normally hyperbolic.

We suppose that $\Phi^{t}: \mathbb{R}^{2 n} \times \mathbb{R} \rightarrow \mathbb{R}^{2 n}$ is a $C^{k}$ smooth flow defined on $\mathbb{R}^{2 n}$ generated by (2.1). We will also need assume the flow to exist for all time, i.e. $t \in \mathbb{R}$. As phase space is not compact, we will need to explicitly discuss the issue of existence of the flow for all time in our examples.

We now state the definition of a NHIM sufficient for our needs.

Definition 1 (NHIM) An $\ell$ dimensional submanifold, $\Lambda$ of $\mathbb{R}^{2 n}(\ell<2 n)$ is said to be a normally hyperbolic invariant manifold for $\Phi^{t}$ if $\Lambda$ is invariant under $\Phi^{t}$ and there exists a splitting of the tangent bundle of $T \mathbb{R}^{2 n}$ into sub-bundles:

$$
T \mathbb{R}^{2 n}=E^{u} \oplus E^{s} \oplus T \Lambda
$$

having the following properties:

Invariance. The sub-bundles are invariant under $D \Phi^{t}$, for all $x \in \Lambda, t \in \mathbb{R}$, i.e.,

$$
\begin{gathered}
v \in E_{x}^{s} \Rightarrow D \Phi^{t}(x)(v) \subset E_{\Phi^{t}(x)}^{s} \quad \text { for all } \quad x \in \Lambda, \quad t \in \mathbb{R}, \\
v \in E_{x}^{u} \Rightarrow D \Phi^{t}(x)(v) \subset E_{\Phi^{t}(x)}^{u} \quad \text { for all } \quad x \in \Lambda, \quad t \in \mathbb{R}, \\
v \in T \Lambda_{x} \Rightarrow D \Phi^{t}(x)(v) \subset T \Lambda_{\Phi^{t}(x)} \quad \text { for all } \quad x \in \Lambda, \quad t \in \mathbb{R}
\end{gathered}
$$

Growth Rates. There exists a constant $C>0$ and rates $0 \leq \beta<\alpha$ such that for all $x \in \Lambda$ we have:

$$
\begin{gathered}
v \in E_{x}^{s} \Rightarrow\left\|D \Phi^{t}(x)(v)\right\| \leq C e^{-\alpha t}\|v\| \quad \text { for all } t \geq 0, \\
v \in E_{x}^{u} \Rightarrow\left\|D \Phi^{t}(x)(v)\right\| \leq C e^{\alpha t}\|v\| \quad \text { for all } \quad t \leq 0, \\
v \in T_{x} \Lambda \Rightarrow\left\|D \Phi^{t}(x)(v)\right\| \leq C e^{\beta|t|}\|v\| \quad \text { for all } t \in \mathbb{R},
\end{gathered}
$$


We now provide some additional background related to the concepts in this definition.

Invariance. Invariance of $\Lambda$ under $\Phi^{t}$ means that for any $x \in \Lambda, \Phi^{t}(x) \in \Lambda$ for all $t \in \mathbb{R}$. We have not required $\Lambda$ to be boundaryless. Making the assumption that $\Lambda$ has no boundary is usual when considering invariance of $\Lambda$, since otherwise one would need to make some sort of assumption preventing trajectories from leaving $\Lambda$ by crossing the boundary of $\Lambda$. We have not required $\Lambda$ to have no boundary since that is not natural for the examples we will consider. Since we will be considering only autonomous Hamiltonian systems the fact that trajectories are prevented from leaving by crossing the boundary of $\Lambda$ is ensured by constancy of the Hamiltonian function, i.e. energy conservation.

Rates. Note the rates $0 \leq \beta<\alpha$. These, along with (2.4a), (2.4b), and (2.4c) encapsulate the idea of normal hyperbolicity, in the sense that the rate of growth or decay of tangent vectors normal to $\Lambda$ under the linearized dynamics dominate the rate of growth or decay of vectors tangent to $\Lambda$ under the linearized dynamics. Note that vectors tangent to $\Lambda$ can still exhibit exponential growth or decay, but the linearized rate must be smaller than that normal to $\Lambda$.

Energy Conservation. The level set of the Hamiltonian,

$$
\Sigma_{E}=\left\{(q, p) \in \mathbb{R}^{2 n} \mid H(q, p)=E\right\}
$$

is a $2 n-1$ dimensional surface (except at possible singular points of the surface) that is invariant under the flow generated by Hamilton's equations. If it is compact and boundaryless, then the flow exists for all time. Generically ${ }^{33}$ the $m$ dimensional manifold $\Lambda$ will intersect 2.5 in a $\ell-1$ dimensional manifold, which we refer to as $\Lambda_{E}$. If $\Lambda$ is normally hyperbolic, then $\Lambda_{E} \equiv \Lambda \cap \Sigma_{E}$ is also normally hyperbolic. Thus, for Hamiltonian systems we will be considering both iso-energetic and non iso-energetic NHIMs.

Dimensionality. We will assume that:

$$
\operatorname{dim} E_{x}^{u}=\operatorname{dim} E_{x}^{s}=m
$$

and it follows from the conditions of Definition 1 that $T_{x} \Lambda, E_{x}^{u}$ and $E_{x}^{s}$ vary continuously with respect to $x$, and therefore the dimensions of $T_{x} \Lambda, E_{x}^{u}$ and $E_{x}^{s}$ are constant 
with respect to $x$. We also have:

$$
\operatorname{dim} T_{x} \Lambda=\ell .
$$

It follows from $(2.2)$ that:

$$
2 n=2 m+\ell .
$$

Hence, $\ell$ is even.

The Need for Bundles: Linearization Characteristics. The 'bundle' concept and terminology, while perhaps unfamiliar, is necessary in our setting since we are considering the linearization about an invariant manifold, which is generally filled with different orbits (as opposed to the more familiar situation of linearizing about a single orbit, such as an equilibrium point or periodic orbit). Consider the unstable bundle, for example. Invariance of this bundle under the linearized flow means that $v \in E_{x}^{u} \Rightarrow D \Phi^{t}(x)(v) \subset E_{\Phi^{t}(x)}^{u} \quad$ for all $\quad x \in \Lambda, \quad t \in \mathbb{R}$. This relation provides an unstable growth direction all along the orbit through $x$, i.e. all along $\Phi^{t}(x), t \in \mathbb{R}$, and enables us to quantify the unstable growth along a given orbit. The (disjoint) union of all of the unstable subspaces over all points in $\Lambda$ gives the unstable bundle over $\Lambda$. Similar consideration hold for the stable bundle over $\Lambda$ and the tangent bundle of $\Lambda$. More details can be found in the general references for NHIMs given earlier.

a. Existence of Stable and Unstable Manifolds. As we have previously mentioned, the definition of NHIM given in Definition 1 specifies conditions on the linearized dynamics about $\Lambda$. These provide sufficient conditions for proving results about the nonlinear dynamics. In particular, the sets of points having the following properties:

$$
\begin{aligned}
& W^{s}(\Lambda)=\left\{y \in \mathbb{R}^{2 n} \mid d\left(\Phi^{t}(y, M) \leq C_{y} e^{-\alpha t} \quad \text { for all } \quad t \geq 0\right\}\right. \\
& W^{u}(\Lambda)=\left\{y \in \mathbb{R}^{2 n} \mid d\left(\Phi^{t}(y, M) \leq C_{y} e^{\alpha t} \quad \text { for all } \quad t \leq 0\right\}\right.
\end{aligned}
$$

can be shown to be $C^{s-1}$ manifolds (for some constant $C_{y}>0$ ), where $s<\min \left\{k, \frac{\alpha}{\beta}\right\}$, where, we recall, $k$ is the degree of differentiability of the flow. Moreover, under the conditions of Definition 1, $\Lambda$ can be shown to be a $C^{s}$ invariant manifold. However, we emphasise again that the conditions of Definition 1 provide sufficient conditions (in terms of linearized dynamics) for the smoothness properties of the manifolds. For example, in the models that 
we consider in Section III A, $\Lambda$ is explicitly known and is infinitely differentiable, regardless of the value of the ratio $\frac{\alpha}{\beta}$. Similarly, the flow will be infinitely differentiable in the models that we consider in Section III A.

b. Persistence Under Perturbation. One of the most important properties of NHIMs is that they persist under $C^{1}$ perturbations. It is important to clearly define the term 'perturbation'. For example, we are considering time independent Hamiltonian systems. Time-dependent erturbations would not satisfy the hypotheses of the perturbation theorem, unless the time-dependence could be recast in a way that the system became time independent, and the resulting system satisfies the hypotheses of the perturbation theorem. Time dependence that is periodic or quasi periodic can be treated in this way, while more general time dependence results in a breakdown of compactness, leading to problems satisfying the hypotheses of the theorem.

c. Sufficient Conditions and 'Breakdown of Normal Hyperbolicity' Definition 1 is a definition of a NHIM. It cannot be concluded that if the conditions of the definition are not satisfied, such as might occur for certain parameter values in a parameterized system, that the NHIM undergoes a 'bifurcation'. Loss of hyperbolicity is a necessary, but not sufficient condition for bifurcation to occur, but the nature of a bifurcation depends on nonlinearity, which is outside the standard requirements of normal hyperbolicity as stated in Definition 1 .

\section{A. The General Form of the Models Under Consideration}

We now consider the general form of a model that encompasses all of the specific examples that we will study. We consider a two DoF Hamiltonian system of the following form:

$$
H\left(q_{1}, p_{1}, q_{2}, p_{2}\right)=H_{1}\left(q_{1}, p_{1}\right)+H_{2}\left(q_{2}, p_{2}\right), \quad\left(q_{1}, p_{1}, q_{2}, p_{2}\right) \in \mathbb{R}^{4}
$$


with associated Hamiltonian vector field:

$$
\begin{aligned}
\dot{q}_{1} & =\frac{\partial H_{1}}{\partial p_{1}}\left(q_{1}, p_{1}\right), \\
\dot{p}_{1} & =-\frac{\partial H_{1}}{\partial q_{1}}\left(q_{1}, p_{1}\right), \\
\dot{q}_{2} & =\frac{\partial H_{2}}{\partial p_{2}}\left(q_{2}, p_{2}\right), \\
\dot{p}_{2} & =-\frac{\partial H_{2}}{\partial q_{2}}\left(q_{2}, p_{2}\right) .
\end{aligned}
$$

Note the particularly simple form of eqs 2.11). It has the form of two separable one degreeof-freedom Hamiltonian systems (hence it is completely integrable). The flow generated by (2.11) has the general form:

$$
\Phi^{t}\left(q_{10}, p_{10}, q_{20}, p_{20}\right)=\left(q_{1}\left(t, q_{10}, p_{10}\right), p_{1}\left(t, q_{10}, p_{10}\right), q_{2}\left(t, q_{20}, p_{20}\right), p_{2}\left(t, q_{20}, p_{20}\right)\right) .
$$

The equation for the (typically) three-dimensional energy surface is given by:

$$
\Sigma_{E}=\left\{\left(q_{1}, p_{1}, q_{2}, p_{2}\right) \in \mathbb{R}^{4} \mid H\left(q_{1}, p_{1}, q_{2}, p_{2}\right)=H_{1}\left(q_{1}, p_{1}\right)+H_{2}\left(q_{2}, p_{2}\right)=E\right\} .
$$

We make the following assumption on the $q_{2}-p_{2}$ component of (2.11).

Assumption 1 At $q_{2}=p_{2}=0$ the system:

$$
\begin{aligned}
& \dot{q}_{2}=\frac{\partial H_{2}}{\partial p_{2}}\left(q_{2}, p_{2}\right), \\
& \dot{p}_{2}=-\frac{\partial H_{2}}{\partial q_{2}}\left(q_{2}, p_{2}\right) .
\end{aligned}
$$

has a hyperbolic equilibrium point. The (positive) eigenvalue of the matrix associated with the linearization of (2.14) about this equilibrium is $\alpha>0$.

Clearly, the set

$$
\Lambda=\left\{\left(q_{1} \cdot p_{1}, q_{2}, p_{2}\right) \mid q_{2}=p_{2}=0\right\}
$$

is a two dimensional invariant manifold in the four dimensional phase space. The intersection of the 3D energy surface with this 2D invariant manifold is given by:

$$
\Lambda_{E}=\Lambda \cap \Sigma_{E}=\left\{\left(q_{1}, p_{1}, q_{2}, p_{2}\right) \in \mathbb{R}^{4} \mid H\left(q_{1}, p_{1}, 0,0\right)=H_{1}\left(q_{1}, p_{1}\right)+H_{2}(0,0)=E\right\},
$$

which is (typically) a one dimensional level set of $H_{1}\left(q_{1}, p_{1}\right)$; an isoenergetic invariant manifold. Now we will show that, under Assumption 1, $\Lambda$ and $\Lambda_{E}$ are both normally hyperbolic invariant manifolds. We therefore need to show that Definition 1 holds for $\Lambda$. 
We begin by computing the linearization of the flow of (2.12) about an arbitrary point on $\Lambda$, which is denoted by $x \equiv\left(q_{1}, p_{1}, 0,0\right)$. First, we transform $H_{2}\left(q_{2}, p_{2}\right)$ to a set of coordinates that facilitates the computations. It follows from Assumption 1 that there exists a linear, symplectic transformation of coordinates, $\left(q_{2}, p_{2}\right) \rightarrow\left(\bar{q}_{2}, \bar{p}_{2}\right)$, such that the quadratic part of $H_{2}$ is "diagonal" in these coordinates, i.e.

$$
H_{2}\left(\bar{q}_{2}, \bar{p}_{2}\right)=H_{2}(0,0)+\alpha \bar{p}_{2} \bar{q}_{2}+H^{3}\left(\bar{q}_{2}, \bar{p}_{2}\right)
$$

where $H^{3}\left(\bar{q}_{2}, \bar{p}_{2}\right)$ is $\mathcal{O}(3)$ (note: there are no linear terms in $H_{2}\left(\bar{q}_{2}, \bar{p}_{2}\right)$ since $\left(q_{2}, p_{2}\right)=$ $\left(\bar{q}_{2}, \bar{p}_{2}\right)=(0,0)$ is an equilibrium point). In these coordinates we can write the Hamiltonian 2.10) in the form:

$$
H\left(q_{1}, p_{1}, \bar{q}_{2}, \bar{p}_{2}\right)=H_{1}\left(q_{1}, p_{1}\right)+H_{2}\left(\bar{q}_{2}, \bar{p}_{2}\right), \quad\left(q_{1}, p_{1}, \bar{q}_{2}, \bar{p}_{2}\right) \in \mathbb{R}^{4}
$$

In these coordinates, the linearisation of the flow has the the following block diagonal form:

$$
D \Phi^{t}(x)=\left(\begin{array}{ccc}
A & 0_{2 \times 2} \\
& e^{\alpha t} & 0 \\
0_{2 \times 2} & 0 & e^{-\alpha t}
\end{array}\right),
$$

where

$$
A=\left(\begin{array}{rr}
\frac{\partial^{2} H_{1}}{\partial q_{1} \partial p_{1}}\left(q_{1}, p_{1}\right) & \frac{\partial^{2} H_{1}}{\partial p_{1}^{2}}\left(q_{1}, p_{1}\right) \\
-\frac{\partial^{2} H_{1}}{\partial q_{1}^{2}}\left(q_{1}, p_{1}\right) & -\frac{\partial^{2} H_{1}}{\partial q_{1} \partial p_{1}}\left(q_{1}, p_{1}\right)
\end{array}\right),
$$

$0_{2 \times 2}$ denotes the $2 \times 2$ matrix of zeros, and we assume that we have transformed the $q_{2}-p_{2}$ coordinates of the lower right hand $2 \times 2$ block so that the flow assumes the diagonal form, which follows from Assumption 1 .

A general tangent vector in $E_{x}^{u}$, where we use the shorthand notation $x=\left(q_{1}, p_{1}, 0,0\right)$, has the form

$$
v=\left(\begin{array}{l}
0 \\
0 \\
1 \\
0
\end{array}\right)
$$

Then we have:

$$
D \Phi^{t}(x)(v)=\left(\begin{array}{c}
0 \\
0 \\
e^{\alpha t} \\
0
\end{array}\right)
$$


which is a tangent vector in $E_{x^{\prime}}^{u}$, where $x^{\prime}=\Phi^{t}(x)$. Since this argument holds for any $x \in \Lambda$ and any $v \in E_{x}^{u}$ it follows that the bundle $E^{u}$ is invariant (i.e. 2.3b holds). Moreover, it follows from (2.22) that the growth rate condition $2.4 \mathrm{~b}$ holds for all vectors in $E^{u}$. A similar argument for the invariance of $E^{s}$ and the growth rate of vectors in $E^{s}$ follows.

Now we turn our attention to the tangent bundle of $\Lambda$. A general vector in $T_{x} \Lambda$ has the form:

$$
v=\left(\begin{array}{l}
a \\
b \\
0 \\
0
\end{array}\right)
$$

Then using 2.19) we have:

$$
D \Phi^{t}(x)(v)=\left(\begin{array}{c}
a^{\prime} \\
b^{\prime} \\
0 \\
0
\end{array}\right)
$$

which is in $T_{x^{\prime}} \Lambda$, where $x^{\prime}=\Phi^{t}(x)$. Here, $a^{\prime}$ and $b^{\prime}$ are functions of $\left(q_{1}, p_{1}\right)$, but the explicit functional form is not important for this argument. Since this argument holds for any $x \in \Lambda$, $v \in T_{x^{\prime}} \Lambda$, it follows that $T \Lambda$ is invariant. What we can not verify at this point is the growth rate condition (2.4c). This will require explicit conditions on the flow on $\Lambda$. The flow on $\Lambda$ can be very general, but normal hyperbolicity will require that $2.4 \mathrm{c}$ ) is satisfied by this flow. This condition will be considered in the specific examples that we analyse in Section III A.

\section{B. Phase space volumes, gap times, and reaction rates}

In this section we briefly review the concepts from classical reaction rate theory that are relevant to the present study. This section is adapted from the paper of Collins et a $\sqrt[34]{ }$ where more background and details can be found.

Points in the 4-dimensional system phase space $\mathcal{M}=\mathbb{R}^{4}$ are denoted $\boldsymbol{z} \equiv\left(p_{1}, p_{2}, q_{1}, q_{2}\right) \equiv$ $(\mathbf{p}, \mathbf{q}) \in \mathcal{M}$. The system Hamiltonian is denoted by $H(\boldsymbol{z})$, and the 3 dimensional energy surface at energy $E, H(\boldsymbol{z})=E$, is denoted $\Sigma_{E} \subset \mathcal{M}$. The corresponding microcanonical phase space density is $\delta(E-H(\boldsymbol{z}))$, and the associated density of states for the complete 
energy surface at energy $E$ is

$$
\rho(E)=\int_{\mathcal{M}} \mathrm{d} \boldsymbol{z} \delta(E-H(\boldsymbol{z})) .
$$

The disjoint regions of phase space ('reactant', $q_{2}<0$, and 'product', $q_{2}>0$ ) separated by the phase space dividing surface $\operatorname{DS}(E)$ are denoted $\mathcal{M}_{ \pm}$; the region of phase space corresponding to $q_{2}>0$ will be denoted by $\mathcal{M}_{+}$, and that corresponding $q_{2}<0$ will be denoted by $\mathcal{M}_{-}$.

The microcanonical density of states for points in region $\mathcal{M}_{+}$is

$$
\rho_{+}(E)=\int_{\mathcal{M}_{+}} \mathrm{d} \boldsymbol{z} \delta(E-H(\boldsymbol{z}))
$$

with a corresponding expression for the density of states $\rho_{-}(E)$ in $\mathcal{M}_{-}$. Since the flow is everywhere transverse to $\mathrm{DS}_{ \pm}(E)$, those phase points in the region $\mathcal{M}_{+}$that lie on trajectories that cross $\operatorname{DS}_{ \pm}(E)$ can be specified uniquely by coordinates $(\widetilde{p}, \widetilde{q}, \psi)$, where $(\widetilde{p}, \widetilde{q}) \in \operatorname{DS}_{+}(E)$ is a point on $\operatorname{DS}_{+}(E)$, specified by 2 coordinates $(\widetilde{p}, \widetilde{q})$, and $\psi$ is a time variable. The point $\boldsymbol{z}(\widetilde{p}, \widetilde{q}, \psi)$ is reached by propagating the initial condition $(\widetilde{p}, \widetilde{q}) \in \operatorname{DS}_{+}(E)$ forward for time $\psi 35136 / 37$. As all initial conditions on $\mathrm{DS}_{+}(E)$ (apart from a set of trajectories of measure zero lying on stable manifolds) will leave the region $\mathcal{M}_{+}$in finite time by crossing $\operatorname{DS}_{-}(E)$, for each $(\widetilde{p}, \widetilde{q}) \in \operatorname{DS}_{+}(E)$, we can define the gap time $s=s(\widetilde{p}, \widetilde{q})$, which is the time it takes for the trajectory to traverse the region $\mathcal{M}_{+}$before entering the region $\mathcal{M}_{\text {. }}$. That is, $\boldsymbol{z}(\widetilde{p}, \widetilde{q}, \psi=s(\widetilde{p}, \widetilde{q})) \in \mathrm{DS}_{-}(E)$. For the phase point $\boldsymbol{z}(\widetilde{p}, \widetilde{q}, \psi)$, we therefore have $0 \leq \psi \leq s(\widetilde{p}, \widetilde{q})$

The coordinate transformation $\boldsymbol{z} \rightarrow(E, \psi, \widetilde{p}, \widetilde{q})$ is canonical $35|38| 39 \mid 40$, so that the phase space volume element is

$$
\mathrm{d}^{4} \boldsymbol{z}=\mathrm{d} E \mathrm{~d} \psi \mathrm{d} \sigma
$$

with $\mathrm{d} \sigma \equiv \mathrm{d} \widetilde{p} \mathrm{~d} \widetilde{q}$ an element of 2 dimensional area on $\mathrm{DS}(\mathrm{E})$.

The magnitude $\phi(E)$ of the flux through dividing surface $\operatorname{DS}_{+}(E)$ at energy $E$ ('directional flux') is given by

$$
\phi(E)=\left|\int_{\mathrm{DS}_{+}(E)} \mathrm{d} \sigma\right|,
$$

where the element of area $\mathrm{d} \sigma$ is precisely the restriction to $\mathrm{DS}(\mathrm{E})$ of the appropriate flux 2 -form $\omega$ corresponding to the Hamiltonian vector field associated with $H(\boldsymbol{z})^{1814144243}$. The 
reactant phase space volume occupied by points initiated on the dividing surface with energies between $E$ and $E+\mathrm{d} E$ is therefore $\mathrm{e}^{35|37| 39|40| 44|45| 46 \mid 47}$

$$
\begin{aligned}
\mathrm{d} E \int_{\mathrm{DS}_{+}(E)} \mathrm{d} \sigma \int_{0}^{s} \mathrm{~d} \psi & =\mathrm{d} E \int_{\mathrm{DS}_{+}(E)} \mathrm{d} \sigma s \\
& =\mathrm{d} E \phi(E) \bar{s}
\end{aligned}
$$

where the mean gap time $\bar{s}$ is defined as

$$
\bar{s}=\frac{1}{\phi(E)} \int_{\mathrm{DS}_{+}(E)} \mathrm{d} \sigma s
$$

and is a function of energy $E$.

\section{Gap time and reactant lifetime distributions}

The gap time distribution, $\mathcal{P}(s ; E)$ is of central interest in unimolecular kinetics $35[48$ : the probability that a phase point on $\operatorname{DS}_{+}(E)$ at energy $E$ has a gap time between $s$ and $s+\mathrm{d} s$ is equal to $\mathcal{P}(s ; E) \mathrm{d} s$. An important idealized gap distribution is the random, exponential distribution

$$
\mathcal{P}(s ; E)=k(E) e^{-k(E) s}
$$

characterized by a single decay constant $k$ (where $k$ depends on energy $E$ ), with corresponding mean gap time $\bar{s}=k^{-1}$. An exponential distribution of gap times is usually taken to be a necessary condition for 'statistical' behavior in unimolecular reactions $35|48| 49|50| 51$.

The lifetime (time to cross the dividing surface $\left.\operatorname{DS}_{-}(E)\right)$ of phase point $\boldsymbol{z}(\widetilde{p}, \widetilde{q}, \psi)$ is $t=$ $s(\widetilde{p}, \widetilde{q})-\psi$, and the corresponding (normalized) reactant lifetime distribution function $\mathbb{P}(t ; E)$ at energy $E$ is $35|48| 49|50| 52|53| 54$

$$
\begin{aligned}
\mathbb{P}(t ; E) & =-\left.\frac{\mathrm{d}}{\mathrm{d} t^{\prime}} \operatorname{Prob}\left(t \geq t^{\prime} ; E\right)\right|_{t^{\prime}=t} \\
& =\frac{1}{\bar{s}} \int_{t}^{+\infty} \mathrm{d} s \mathcal{P}(s ; E)
\end{aligned}
$$

where the fraction of interesting (reactive) phase points having lifetimes between $t$ and $t+\mathrm{d} t$ is $\mathbb{P}(t ; E) \mathrm{d} t$. It is often useful to work with the unnormalized lifetime distribution $F$, where $F(t ; E) \equiv \bar{s} \mathbb{P}(t ; E)$. 
Equation 2.32a gives the general relation between the lifetime distribution and the fraction of trajectories having lifetimes greater than a certain value for arbitrary ensembles 52153154 . Note that an exponential gap time distribution (2.31) implies that the reactant lifetime distribution $\mathbb{P}(t ; E)$ is also exponential $\frac{35|48| 49|52| 53 \mid 54}{\text {; }}$ both gap and lifetime distributions for realistic molecular potentials have been of great interest since the earliest days of trajectory simulations of unimolecular decay, and many examples of non-exponential lifetime distributions have been found $36|52| 53|54| 55|56| 57|58| 59$. 


\section{MODEL DYNAMICS: DIVIDING SURFACES, FLUX, BREAKDOWN OF NORMAL HYPERBOLICITY, AND GAP TIME DISTRIBUTIONS}

In this section we describe our two model systems. Each model has NHIMs, and we describe the normal hyperbolicity properties of the NHIMs as a function of energy and other parameters. We then compute the flux through the associated DS as well as the gap time distribution, and we discuss the behaviour of these quantities in relation to the property of normal hyperbolicity.

\section{A. The Models}

The two models to be studied are particular examples of the general class of models described in Section $\amalg$ A.

\section{Example 1. Uncoupled Simple Pendulum and Symmetric Double-Well}

Our first example will consist of an integrable system whose Hamiltonian is the sum of the Hamiltonian of a simple pendulum and the Hamiltonian of a symmetric double-well:

$$
H=\frac{p_{1}^{2}}{2}-\alpha_{1}^{2} \cos q_{1}+\frac{p_{2}^{2}}{2}-\frac{\alpha_{2}}{2} q_{2}^{2}+\frac{1}{4} q_{2}^{4}=H_{1}\left(q_{1}, p_{1}\right)+H_{2}\left(q_{2}, p_{2}\right),
$$

with associated equations of motion:

$$
\begin{aligned}
& \dot{q}_{1}=\frac{\partial H}{\partial p_{1}}=p_{1} \\
& \dot{p}_{1}=-\frac{\partial H}{\partial q_{1}}=-\alpha_{1}^{2} \sin q_{1} \\
& \dot{q}_{2}=\frac{\partial H}{\partial p_{2}}=p_{2} \\
& \dot{p}_{2}=-\frac{\partial H}{\partial q_{2}}=\alpha_{2} q_{2}-q_{2}^{3}
\end{aligned}
$$

The phase space structure for these two, uncoupled, one degree-of-freedom Hamiltonian systems is illustrated in Fig. 1. Since the trajectories of (3.2) can be solved analytically, for any initial condition, it can be seen explicitly that the flow generated by (3.2) exists for all time.

Following 2.15), the two dimensional non-isoenergetic surface:

$$
\Lambda \equiv\left\{\left(q_{1}, p_{1}, q_{2}, p_{2}\right) \in \mathbb{R}^{4} \mid q_{2}=p_{2}=0\right\}
$$


is an invariant manifold for (3.6). For $\alpha_{2}>0$, tangent vectors normal to $\Lambda$ experience exponential growth and decay under the linearised dynamics. However, we cannot claim that it is a NHIM according to definition 1. Whether or not this is in fact the case depends on the dynamics on $\Lambda$. Following (2.16), the intersection of the three dimensional energy surface (3.1) with 3.3 is given by:

$$
\Lambda_{E} \equiv\left\{\left(q_{1}, p_{1}, q_{2}, p_{2}\right) \in \mathbb{R}^{4} \mid \frac{p_{1}^{2}}{2}-\alpha_{1}^{2} \cos q_{2}=E, q_{2}=p_{2}=0\right\}
$$

Hence, the dynamics on $\Lambda$ is described by the dynamics of a (symmetric) two well potential, and an orbit of the pendulum with energy $E$ defines $\Lambda_{E}$. We note that:

- $q_{1}=p_{1}=0$ is an elliptic equilibrium point of the pendulum with energy $E=-\alpha_{1}^{2}$.

- Surrounding the elliptic fixed point is a family of periodic orbits ('librations') whose energies increase monotonically from $E=-\alpha_{1}^{2}$ to $E=\alpha_{1}^{2}$.

- $q_{1}=\pi, p_{1}=0$ is a saddle point with energy $E=\alpha_{1}^{2}$ that is connected by a pair of homoclinic orbits.

- Outside the pair of homoclinic orbits are two families of periodic orbits ('rotations') whose actions increase monotonically with energy, from $E=\alpha_{1}^{2}$.

The phase space structure associated with the simple pendulum on $\Lambda$ is shown in Fig. 1 . We note that homoclinic orbits have zero frequency. Hence, for one DoF Hamiltonian systems, they are examples of the phenomenon of resonance. The presence of homoclinic orbits therefore allows us to examine the role of resonance in the breakdown of normal hyperbolicity explicitly.

In our model we take 'reaction' to correspond to a change of sign of the coordinate $q_{2}$. Hence, the energy of reacting trajectories in the $q_{2}=p_{2}$ coordinates must be greater than that of the saddle point at $q_{2}=p_{2}=0$, i.e., the energy must be greater than zero. Hence, the total energy must be such that the energy in the $q_{2}-p_{2}$ subsystem is greater than zero. 


\section{Example 2. Two Uncoupled Symmetric Double-Wells}

The second example consists of two uncoupled symmetric double-well Hamiltonians:

$$
\begin{aligned}
H & =\frac{p_{1}^{2}}{2}-\frac{\alpha_{1}}{2} q_{1}^{2}+\frac{1}{4} q_{1}^{4}+\frac{p_{2}^{2}}{2}-\frac{\alpha_{2}}{2} q_{2}^{2}+\frac{1}{4} q_{2}^{4} \\
& =H_{1}\left(q_{1}, p_{1}\right)+H_{2}\left(q_{2}, p_{2}\right),
\end{aligned}
$$

with associated Hamiltonian vector field:

$$
\begin{aligned}
& \dot{q}_{1}=\frac{\partial H}{\partial p_{1}}=p_{1}, \\
& \dot{p}_{1}=-\frac{\partial H}{\partial q_{1}}=\alpha_{1} q_{1}-q_{1}^{3}, \\
& \dot{q}_{2}=\frac{\partial H}{\partial p_{2}}=p_{2}, \\
& \dot{p}_{2}=-\frac{\partial H}{\partial q_{2}}=\alpha_{2} q_{2}-q_{2}^{3} .
\end{aligned}
$$

Again, the trajectories of (3.6) can be solved analytically for any initial condition, and the flow generated by (3.6) exists for all time.

The discussion of the phase space structure is similar to that for Example 1 above. Following (2.15), the two dimensional non-isoenergetic surface:

$$
\Lambda \equiv\left\{\left(q_{1}, p_{1}, q_{2}, p_{2}\right) \in \mathbb{R}^{4} \mid q_{2}=p_{2}=0\right\}
$$

is an invariant manifold for (3.6). For $\alpha_{2}>0$, tangent vectors normal to $\Lambda$ experience exponential growth and decay under the linearised dynamics. Again, whether or not $\Lambda$ is a NHIM according to definition 1 depends on the dynamics on $\Lambda$. Following (2.16), the intersection of the three dimensional energy surface (3.5) with (3.7) is given by:

$$
\Lambda_{E} \equiv\left\{\left(q_{1}, p_{1}, q_{2}, p_{2}\right) \in \mathbb{R}^{4} \mid \frac{p_{1}^{2}}{2}-\frac{\alpha_{1}}{2} q_{1}^{2}+\frac{1}{4} q_{1}^{4}=E, q_{2}=p_{2}=0\right\} .
$$

Hence, the dynamics on $\Lambda$ is described by the dynamics of a symmetric two well potential, and an orbit of the symmetric two well potential with energy $E$ defines $\Lambda_{E}$. We note that:

- $q_{1}=p_{1}=0$ is a saddle point of the symmetric two well potential with energy $E=0$.

- $q_{1}= \pm \sqrt{\alpha_{1}}, p_{1}=0$ are elliptic equilibrium points with energy $-\frac{1}{4} \alpha_{1}^{2}$.

- Surrounding each elliptic equilibrium point is a family of periodic orbits whose energies increase monotonically away from the equilibrium point. At $E=0$ the periodic orbits merge into a pair of homoclinic orbits that connect the saddle point at the origin. 
- As the energy increases from zero there is a family of (symmetric) periodic orbits that surround both potential wells whose actions increase monotonically with energy.

The phase space structure associated with the symmetric double well on $\Lambda$ is shown in Fig. 2.

As in the previous model, 'reaction' is associated with a change of sign of the $q_{2}$ coordinate. Hence, the energy of reacting trajectories in the $q_{2}-p_{2}$ coordinates must be greater than the energy of the saddle point at $q_{2}=p_{2}=0$, i.e. the energy must be greater than zero. The total energy must therefore be such that the energy in the $q_{2}-p_{2}$ component of the model is greater than zero.

\section{B. Dividing Surfaces and Flux}

For each of our model Hamiltonians we can construct a dividing surface in phase space having the (local) no-recrossing property. The codimension one non-isoenergetic surface defined by $q_{2}=0$ (locally) divides the phase space into two regions: one associated with the regions defined by $q_{2}>0$ and the other associated with the region defined by $q_{2}<0$. The dividing surface restricted to a three dimensional fixed energy surface $H\left(q_{1}, p_{1}, q_{2}, p_{2}\right)=$ $H_{1}\left(q_{1}, p_{1}\right)+H_{2}\left(q_{2}, p_{2}\right)=E$ is given by:

$$
D S(E)=\left\{\left(q_{1}, p_{1}, q, p_{2}\right) \mid q_{2}=0, H=H_{1}\left(q_{1}, p_{1}\right)+\frac{p_{2}^{2}}{2}=E\right\} .
$$

This dividing surface has two halves:

$$
D S_{+}(E)=\left\{\left(q_{1}, p_{1}, q_{2}, p_{2}\right) \mid q_{2}=0, H=H_{1}\left(q_{1}, p_{1}\right)+\frac{p_{2}^{2}}{2}=E, p_{2}>0\right\}
$$

and

$$
D S_{-}(E)=\left\{\left(q_{1}, p_{1}, q_{2}, p_{2}\right) \mid q_{2}=0, H=H_{1}\left(q_{1}, p_{1}\right)+\frac{p_{2}^{2}}{2} \bar{p}_{2}^{2}=E, p_{2}<0\right\} .
$$

These two halves meet at an invariant manifold:

$$
\Lambda_{E}=\left\{\left(q_{1}, p_{1}, q_{2}, p_{2}\right) \mid q_{2}=0, H=H_{1}\left(q_{1}, p_{1}\right)=E, p_{2}=0\right\}
$$

The nature of this invariant manifold (especially the normal hyperbolicity property) depends on $E$, the dynamics on $\Lambda_{E}$, and a comparison of the linearised growth rates normal and 
tangent to $E$, as we will see for our examples. In particular, we will examine the interrelation between these characteristics.

We now describe conditions under which $D S_{+}(E)$ and $D S_{-}(E)$ are surfaces having the no (local) re-crossing property (and we will verify these conditions in our particular examples). These surfaces are defined by $q_{2}=0$. Therefore points on these surfaces leave if:

$$
\dot{q}_{2}=p_{2} \neq 0
$$

and therefore it follows immediately from their definition that (3.10a) and (3.10b) have the (local) 'no-recrossing' property.

We denote the directional flux across these hemispheres by $\phi_{+}(E)$ and $\phi_{-}(E)$, respectively, and note that $\phi_{+}(E)+\phi_{-}(E)=0$. The magnitude of the flux is $\left|\phi_{+}(E)\right|=\left|\phi_{-}(E)\right| \equiv \phi(E)$. The magnitude of the flux and related quantities are central to the theory of reaction rates, as we described in Section $\amalg$ B.

\section{Example 1. Simple pendulum plus Symmetric Double-Well}

a. Dividing surface. In Figure 3 we plot the dividing surface (i.e. (3.9)) for three different energies: $E=0.5$ (energy below the separatrices of the pendulum), $E=\alpha_{1}^{2}=0.8^{2}$ (energy equal to the energy of the separatrices of the pendulum), and $E=1$ (energy larger than the energy of the separatrices of the pendulum). We see that the DS undergoes a bifurcation as the energy passes through the energy of the separatrix. A central question is whether or not this bifurcation has any effect on quantities that are important for quantifying reaction dynamics, such as flux and gap times.

b. Flux across the DS. By Stokes theorem, the directional flux across half the DS, at energy $E$, is the area enclosed by the invariant manifold $\Lambda_{E}$ on the $\left(q_{1}, p_{1}\right)$ plane. This area is just the integral $A=\oint p_{1} d q_{1}$ and is related to the action variable of the simple pendulum

by $J=\frac{1}{2 \pi} \oint p_{1} d q_{1}$ so that we have the relation $A=2 \pi J$. For the simple pendulum there are three different cases to consider for the calculation of the action integral: the librations, the separatrices, and the rotations. The computation of these integrals for the different cases is 
reviewed in the appendix A. Here we just note the results:

$$
\begin{aligned}
& J_{l}(E)=\frac{8 \alpha_{1}}{\pi}\left[\mathcal{E}_{1}\left(k_{l}\right)+k_{l}^{2} \mathcal{K}_{1}\left(k_{l}\right)-\mathcal{K}_{1}\left(k_{l}\right)\right] \\
& J_{s}(E)=\frac{4 \alpha_{1}}{\pi} \\
& J_{r}(E)=\frac{4 \alpha_{1}}{k_{r} \pi} \mathcal{E}_{1}\left(k_{r}\right)
\end{aligned}
$$

where the subscripts $l, s$ and $r$ denote libration, separatrix and rotation, respectively. $\mathcal{K}_{1}$ and $\mathcal{E}_{1}$ denote the complete elliptic integrals of the first and second kind, respectively and $k_{l}$ and $k_{r}$ are the moduli of the complete elliptic integrals, and are functions of the energy $E$ :

$$
\begin{aligned}
& k_{l}(E)=\sin \left[-\frac{1}{2} \cos ^{-1}\left(\frac{E}{\alpha_{1}^{2}}\right)\right] \\
& k_{r}(E)=\sqrt{\frac{2 \alpha_{1}^{2}}{E+\alpha_{1}^{2}}}
\end{aligned}
$$

With these expressions in hand we obtain expressions for the directional flux $\phi(E)$ across half the DS:

$$
\begin{aligned}
\phi_{l}(E) & =16 \alpha_{1}\left[\mathcal{E}_{1}\left(k_{l}\right)+k_{l}^{2} \mathcal{K}_{1}\left(k_{l}\right)-\mathcal{K}_{1}\left(k_{l}\right)\right] \\
\phi_{s}(E) & =16 \alpha_{1} \\
\phi_{r}(E) & =\frac{16 \alpha_{1}}{k_{r}} \mathcal{E}_{1}\left(k_{r}\right)
\end{aligned}
$$

For the separatrix and the rotation cases an extra factor 2 appears because one must count the area enclosed by the curves having positive and negative momentum. The graph of the flux $\phi(E)$ is shown in Figure 4.

\section{Example 2. Uncoupled Symmetric Double-Wells}

a. Dividing surface. In Figure 5 we plot the dividing surface (i.e., the surface (3.9) for three different energies: $E=-0.5$ (energy below the separatrices of the two well potential), $E=0$ (the energy of the separatrices of the two well potential), and $E=1$ (energy larger than the energy of the separatrices of the two well potential). As for example 1, the DS undergoes a bifurcation as the energy passes through the energy of the separatrix. 
b. Flux across the DS. As for Example 1, the directional flux across half the DS is the area enclosed by the invariant manifold $\Lambda_{E}$ on the $\left(q_{1}, p_{1}\right)$ plane. This area is the integral $A=\oint p_{1} d q_{1}$ and is related to the action variable of the symmetric double-well by $A=2 \pi J$. For the symmetric double-well there are three different cases for the calculation of the action integral depending on the energy being less, greater or equal to the energy of the saddle point. The integrals for the different cases are (cf. appendix B):

$$
\begin{aligned}
J_{1}(E) & =\frac{b \delta}{3 \sqrt{2} \pi k_{1}^{2}}\left[\left(2-k_{1}^{2}\right) \mathcal{E}_{1}\left(k_{1}\right)+\left(2 k_{1}^{2}-2\right) \mathcal{K}_{1}\left(k_{1}\right)\right] \\
J_{2}(E) & =\frac{2 \alpha_{2}^{3 / 2}}{3 \pi} \\
J_{3}(E) & =\frac{\sqrt{2 \delta} b^{2}}{3 k_{3}^{2} \pi}\left[\left(2 k_{3}^{2}-1\right) \mathcal{E}_{1}\left(k_{3}\right)+\left(1-k_{3}^{2}\right) \mathcal{K}_{1}\left(k_{3}\right)\right]
\end{aligned}
$$

where the subscript 1, 2 and 3 refers to the three different regimes of energy, that is, energy below, and above the energy of the saddle point, respectively. In theses expressions $a$ and $b$ are roots of the equation $p_{1}=0\left(c f\right.$. Appendix $\mathrm{B}$ ) and $\delta=b^{2}-a^{2}$. All these 3 quantities are functions of the energy. As for the case of the pendulum, the moduli $k_{1}$ and $k_{3}$ of the elliptic integrals are functions of the energy:

$$
\begin{aligned}
a(E) & =\sqrt{\alpha_{1}-\sqrt{\alpha_{1}^{2}+4 E}} \\
b(E) & =\sqrt{\alpha_{1}+\sqrt{\alpha_{1}^{2}+4 E}} \\
k_{1}(E) & =\sqrt{\frac{\delta(E)}{b^{2}(E)}} \\
k_{3}(E) & =\sqrt{\frac{b^{2}(E)}{\delta(E)}} .
\end{aligned}
$$

The directional flux $\phi(E)$ across half of the DS is then given by:

$$
\begin{aligned}
\phi_{1}(E) & =\frac{4 b \delta}{3 \sqrt{2} k_{1}^{2}}\left[\left(2-k_{1}^{2}\right) \mathcal{E}_{1}\left(k_{1}\right)+\left(2 k_{1}^{2}-2\right) \mathcal{K}_{1}\left(k_{1}\right)\right] \\
\phi_{2}(E) & =\frac{8 \alpha_{2}^{3 / 2}}{3} \\
\phi_{3}(E) & =\frac{2 \sqrt{2 \delta} b^{2}}{3 k_{3}^{2}}\left[\left(2 k_{3}^{2}-1\right) \mathcal{E}_{1}\left(k_{3}\right)+\left(1-k_{3}^{2}\right) \mathcal{K}_{1}\left(k_{3}\right)\right] .
\end{aligned}
$$

A plot of the flux $\phi(E)$ as a function of energy $E$ is shown in Figure 6 . 


\section{Gap Time Distributions}

In this subsection we compute the gap time distribution of our two examples. Before proceeding to the calculation of the gap time distribution, we need to determine the gap time for reactant trajectories. In our two examples the determination of the gap times of reacting orbits is straightforward since the gap time is just half of the period of the periodic orbits in the double-well degree-of-freedom $\left(q_{2}-p_{2}\right)$ for which the energy is greater than the energy of the saddle point $\left(q_{2}=0, p_{2}=0\right)$. The period of these periodic orbits as a function of the energy is given by:

$$
T\left(E_{2}\right)=\frac{4 \sqrt{2}}{\sqrt{\delta\left(E_{2}\right)}} \mathcal{K}\left(k_{3}\left(E_{2}\right)\right),
$$

where $E_{2}$ stands for energy in the independent second degree-of-freedom, and the total energy of the two, uncoupled one degree-of-freedom systems is denoted by $E_{T}=E_{1}+E_{2}$. The gap time as a function of the energy $E_{2}$ is then given by:

$$
s\left(E_{2}\right)=\frac{2 \sqrt{2}}{\sqrt{\delta\left(E_{2}\right)}} \mathcal{K}\left(k_{3}\left(E_{2}\right)\right),
$$

For clarity here we will use the notations $I$ for the action associated with the first degreeof-freedom (pendulum for example one and double-well for example 2) and $J$ for the action associated with the second degree-of-freedom. Because of the direct relation between the energies of the different degrees-of-freedom and the actions associated with these degreesof-freedom, at constant energy we have:

$$
J=J\left(E_{T}, I\right)
$$

so that we can formally write the gap time as a function of the total energy and the action $I$ : $s\left(E_{2}\right)=s\left(E_{T}, I\right)$. Again at constant $E_{T}$, the number of trajectories having $s<\bar{s}=\bar{s}\left(E_{T}, \bar{I}\right)$ is proportional to the area enclosed by the curve $I=\bar{I}$ in the $\left(q_{1}-p_{1}\right)$ plane, that is to say, it equals $2 \pi \bar{I}$. The number of trajectories $n$ for which the gap time $s$ has the property that $\bar{s} \leq s \leq \bar{s}+\Delta s$ is:

$$
n=2 \pi[(\bar{I}+\Delta I)-\bar{I}]=2 \pi \frac{\partial I}{\partial s} \Delta s
$$


The gap time distribution is now given by:

$$
\begin{aligned}
P\left(s ; E_{T}\right) & =\frac{n}{\Delta s} \\
& =2 \pi \frac{\partial I}{\partial s} \\
& =\frac{2 \pi}{\left.\frac{\partial s}{\partial I}\right|_{E_{T}}} .
\end{aligned}
$$

Figure 7 shows the gap time distribution for both examples.

Figure 7 shows that the gap time distributions for both examples possess a singularity. This singularity arises from the vanishing of the derivative of the action $I$ with respect to the gap time $s$, which is equivalent to the singularity of the derivative of this action with respect to the energy $E_{1}$. This singularity appears because the action variable $I$ is a piecewise defined function with respect to the energy $E_{1}$. Whereas the action $I$ is a continuous function with respect to the energy $E_{1}$, its derivative (the inverse of the associated frequency) diverges at the separatrix (homoclinic orbits), which is exactly where bifurcation occurs. Interpreted geometrically, we see that just below the bifurcation the rate of growth of the area enclosed by the periodic orbits in the $\left(q_{1}-p_{1}\right)$ plane increases to infinity as we get closer to the homoclinic orbit. In the same way, just after the bifurcation this growth rate decreases from infinity. The appearance of homoclinic orbits is manifested in the flux $\phi\left(E_{T}\right)$, which is directly related to the action $I$, through the appearance of an inflexion point at the energy of the bifurcation.

\section{Loss of normal hyperbolicity and its consequences on the dynamics of reaction.}

In this section we will turn our attention to the question of normal hyperbolicity of the isoenergetic invariant manifold, which forms the boundary of the isoenergetic dividing surface. For both examples $\Lambda_{E}$ is either a periodic orbit or the union of homoclinic orbits and the saddle points that they connect. The two dimensional non-isoenergetic invariant manifold is defined by $q_{2}=p_{2}=0$, which is a hyperbolic saddle point with associated growth rate $\alpha_{2}$. For $\alpha_{2}>0$ tangent vectors normal to $\Lambda$ experience exponential growth and decay under the linearised dynamics. Hence one way that normal hyperbolicity could be lost is for $\alpha_{2}$ to go from positive to negative. However, this possibility is not interesting from the point of view of reaction dynamics since this would destroy the reactive (double well) nature of the problem. Hence, we will require $\alpha_{2}>0$. 
The more interesting situation is the violation of the growth condition $2.4 \mathrm{c})$ in the definition of a NHIM (Definition 1). When $\Lambda_{E}$ is a periodic orbit, this growth rate is zero, and $\Lambda_{E}$ is a NHIM. When $\Lambda_{E}$ is a union of homoclinic orbits and the saddle points that they connect, whether or not $\Lambda_{E}$ satisfies the growth rate conditions of Definition 1 depends on the nature of $\alpha_{1}$ and $\alpha_{2}$. In particular, for Example $1 \pm \alpha_{1}$ are the eigenvalues associated with the saddle point on the $q_{1}-p_{1}$ plane and for Example $2 \pm \sqrt{\alpha_{1}}$ are the eigenvalues associated with the saddle point in the $q_{1}=p_{1}$ plane.

We therefore have the following situations:

Example 1: For $\alpha_{1}>\sqrt{\alpha_{2}}$ the growth rate conditions of Definition 1 are not satisfied, and $\Lambda_{E}$ is not a NHIM.

Example 2: For $\alpha_{1}>\alpha_{2}$ the growth rate conditions of Definition 1 are not satisfied, and $\Lambda_{E}$ is not a NHIM.

We now examine the consequences of this 'loss of normal hyperbolicity' in more detail.

\section{Consequences of loss of normal hyperbolicity}

We imagine the parameters $\alpha_{1}>\sqrt{\alpha_{2}}$ fixed for example 1 and $\alpha_{1}>\alpha_{2}$ for example 2, and we vary the energy in such a way that we pass through the energy value corresponding to the homoclinic orbits and saddle points. With $\alpha_{1}$ and $\alpha_{2}$ fixed as above, at this energy value the conditions of Definition 1 do not hold. This situation could be referred to as 'loss of normal hyperbolicity'. We examine the implication of this loss of normal hyperbolicity for the quantities that we have computed for our two examples.

\section{The Dividing Surface and the (Directional) Flux Through the Dividing Surface.}

The dividing surface, as a function of energy, is shown in Figure 3 for example 1 and in Figure 5 for example 2. While in both examples the geometry of the surface undergoes a qualitative change is we pass through the bifurcation, we are still able to define a dividing surface having the no recrossing property as we pass through the bifurcation, i.e., even as the NHIM experiences a loss of normal hyperbolicity (as just defined). 
The directional flux, as a function of energy, is shown in Figure 4 for example 1 and in Figure 6 for example 2. For both examples we see that the flux varies continuously as a function of the energy, even as we pass through the bifurcation, i.e., even as the NHIM experiences a loss of normal hyperbolicity.

The Gap Time Distribution. From Figure 7 we have already noted note that the gap time distributions for both examples possess a singularity. This singularity is associated with the existence of a homoclinic orbit in the DS, and is not related to the loss of normal hyperbolicity of the NHIM. 


\section{CONCLUSIONS AND OUTLOOK}

In this paper we have studied the breakdown of normal hyperbolicity and its consequences for quantities related to reaction dynamics; in particular, the dividing surface, the flux through the dividing surface, and the gap time distribution.

Our approach is to study these questions using simple, two degree-of-freedom Hamiltonian models for which calculations for the different geometrical and dynamical quantities can be carried out exactly. For our examples, we showed that resonances (homoclinic orbits) within the normally hyperbolic invariant manifold may, or may not, lead to 'loss of normal hyperbolicity'. Moreover, we showed that for our examples the onset of such resonances results in a change in topology of the dividing surface, but it does not affect our ability to define a dividing surface (DS), and that the flux through the DS varies continuously with energy, even as the energy is varied in such a way that normal hyperbolicity is lost. For both our examples we have shown that the gap time distribution exhibits a signature singularity at energies corresponding to emergence of a homoclinic orbit in the DS, but these singularities are not associated with loss of normal hyperbolicity.

\section{Acknowledgments}

FM, PC, and SW acknowledge the support of the Office of Naval Research (Grant No. N00014-01-1-0769) and the Leverhulme Trust. 


\section{Appendix A: Action variables for the simple pendulum}

In this appendix we review the compution of action variables for the integrable simple pendulum system. The Hamiltonian of the simple pendulum is:

$$
H=\frac{p^{2}}{2}-\alpha_{1}^{2} \cos q
$$

In order to determine the action variable for this system we have to consider three different cases: libration $\left(E<\alpha_{1}^{2}\right)$, separatrix $\left(E=\alpha_{1}^{2}\right)$, and rotation $\left(E>\alpha_{1}^{2}\right)$.

\section{Libration}

The action for this case is given by:

$$
J_{l}=\frac{1}{2 \pi} \oint p d q
$$

For this case the system doesn't have enough energy to cover the full range of the angle $q$ and there should be two turning points where the momentum vanishes, $p=0$. Let $q_{0}$ be the positive value of $q$ at the turning point which depends on the energy:

$$
q_{0}=\cos ^{-1}\left(-\frac{E}{\alpha_{1}^{2}}\right) \text {. }
$$

The momentum $p$ can be expressed as a function of $q_{0}$ and $q$ :

$$
p=\sqrt{2 \alpha_{1}^{2}\left(\cos q-\cos q_{0}\right)} .
$$

Substituting this expression into the eq. $\mathrm{A} 2$ we obtain the integral:

$$
J_{l}=\frac{4 \alpha_{1}}{\pi} \int_{0}^{q_{0}} \sqrt{\sin ^{2}\left(\frac{q_{0}}{2}\right)-\sin ^{2}\left(\frac{q}{2}\right)} d q .
$$

After an appropriate change of variable we can put the former integral into a form which makes it resemble an elliptic integral:

$$
J_{l}=\frac{8 \alpha_{1} k_{l}^{2}}{\pi} \int_{0}^{\pi / 2} \frac{\cos ^{2} \phi}{\sqrt{1-k_{l}^{2} \sin ^{2} \phi}} d \phi
$$

where the modulus $k_{l}$ of the elliptic integral is given by $k_{l}=\sin \left(\frac{q_{0}}{2}\right)$. This integral can be expressed in terms of complete elliptic integrals as follows:

$$
J_{l}=\frac{8 \alpha_{1}}{\pi}\left[\mathcal{E}_{1}\left(k_{l}\right)+k_{l}^{2} \mathcal{K}_{1}\left(k_{l}\right)-\mathcal{K}_{1}\left(k_{l}\right)\right]
$$

where $\mathcal{K}_{1}$ and $\mathcal{E}_{1}$ stand for the complete elliptic integrals of the first and second kinds respectively $\sqrt{60}$. 


\section{Rotation}

For this case the system has enough energy to cover the full range of the angle $q$ and there is no point where the momentum vanishes. The momentum is given by:

$$
p=\sqrt{2\left(E+\alpha_{1}^{2} \cos q\right)}
$$

The corresponding action integral is:

$$
J_{r}=\frac{1}{2 \pi} \int_{0}^{2 \pi} \sqrt{2\left(E+\alpha_{1}^{2} \cos q\right)} d q .
$$

Again using a change of variables we can transform this integral to:

$$
J_{r}=\frac{2 \alpha_{1}}{k_{r} \pi} \int_{0}^{\pi} \sqrt{1-k_{r}^{2} \sin ^{2} \phi} d \phi,
$$

where the modulus $k_{r}$ is given by $k_{r}=\sqrt{\frac{2 \alpha_{1}^{2}}{E+\alpha_{1}^{2}}}$. This integral is just twice the complete elliptic integral of the second kind, so that the action for the rotation case is:

$$
J_{r}=\frac{4 \alpha_{1}}{k_{r} \pi} \mathcal{E}_{1}\left(k_{r}\right)
$$

\section{The separatrix case}

For the separatrix case we can repeat the same kind of calculations as for the rotation case. Noting that for this case we have $k_{r}=1$ and $\mathcal{E}_{1}(1)=1$ the action for the separatrix case is just:

$$
J_{s}=\frac{4 \alpha_{1}}{\pi}
$$

\section{Appendix B: Action variables for the symmetric double-well}

In this appendix we compute action variables for the integrable symmetric double-well system. The Hamiltonian of the system is:

$$
H=\frac{p^{2}}{2}-\alpha_{2} \frac{q^{2}}{2}+\frac{q^{4}}{4}
$$

In order to determine the action variable of this system we have to consider three different cases distinguished by the value of the energy $E: E<0$, the separatrix case where $E=0$, and $E>0$. 


\section{Case $E<0$.}

For this case the energy is below the saddle point energy, $E=0$. Trajectories are confined to one of the two wells. Because the double-well is symmetric the action variable is the same for the motions in either well.From eq. (B1) we can write the momentum $p$ as a function of the energy and $q$ :

$$
p=\sqrt{2 E+\alpha_{2} q^{2}-\frac{q^{4}}{2}} .
$$

The associated action integral is:

$$
J_{1}=\frac{1}{2 \pi} \oint p d q=\frac{1}{2 \pi} 2 \int_{a}^{b} \sqrt{2 E+\alpha_{2} q^{2}-\frac{q^{4}}{2}} d q,
$$

where $a$ and $b$ are the two roots of the equation $p=0$ for the well situated situated on the side where $q>0$ for example and with $a<b$.

Using a change of variable we can rearrange the result into the form:

$$
J_{1}=\frac{b \delta k_{1}^{2}}{\sqrt{2} \pi} \int_{0}^{\pi / 2} \frac{\sin ^{2} \theta \cos ^{2} \theta}{\sqrt{1-k_{1}^{2} \sin ^{2} \theta}} d \theta
$$

where $\delta=b^{2}-a^{2}$ and $k_{1}=\sqrt{\frac{\delta}{b^{2}}}$. The latter integral can be evaluated by parts and we finally obtain:

$$
J_{1}=\frac{b \delta}{3 \sqrt{2} \pi k_{1}^{2}}\left[\left(2-k_{1}^{2}\right) \mathcal{E}_{1}\left(k_{1}\right)+\left(2 k_{1}^{2}-2\right) \mathcal{K}_{1}\left(k_{1}\right)\right]
$$

\section{Case $E>0$.}

For this case the energy is above the saddle point energy. In this case the equation $p=0$ has only two roots situated symmetrically with respect to the coordinate origin $q=0$. Denoting the two roots $a$ and $b, a<b$, the action integral is:

$$
J_{3}=\frac{1}{2 \pi} \oint p d q=\frac{4}{2 \pi} \int_{0}^{b} p d q,
$$

Using an appropriate change of variable we can transform this integral to the form:

$$
J_{3}=\frac{\sqrt{2 \delta} b^{2}}{\pi} \int_{0}^{\pi / 2} \sqrt{1-k_{3}^{3} \sin ^{2} \theta} \sin ^{2} \theta d \theta,
$$

where $\delta=b^{2}-a^{2}$ and $k_{3}=\sqrt{\frac{b^{2}}{\delta}}$. Inetgrals of this type are tabulated (see for example ref. 60) and one gets the result:

$$
J_{3}=\frac{\sqrt{2 \delta} b^{2}}{3 \pi k_{3}^{2}}\left[\left(2 k_{3}^{2}-1\right) \mathcal{E}_{1}\left(k_{3}\right)+\left(1-k_{3}^{2}\right) \mathcal{K}_{1}\left(k_{3}\right)\right] .
$$




\section{Case $E=0$.}

The action in this case is obtained by setting $a=0, b=\sqrt{2 \alpha_{2}}, \delta=b^{2}$ and $k_{3}=1$ in the previous case, and noting also that instead of having a factor 4 in eq. (B6) we have here a factor 2 . Taking into account the fact that $\mathcal{E}_{1}(1)=1$ we get for the separatrix action:

$$
J_{2}=\frac{2 \alpha_{2}^{3 / 2}}{3 \pi} .
$$


* frederic.mauguiere@bristol.ac.uk

$\dagger$ gse1@cornell.edu

$\ddagger$ stephen.wiggins@mac.com

1 P. Pechukas. Transition State Theory. Ann. Rev. Phys. Chem., 32:159-177, 1981.

2 E. Pollak and P. Talkner. Reaction rate theory: What it was, where it is today, and where is it going? Chaos, 15:026116, 2005.

3 K. J. Laidler and M. C. King. The development of transition state theory. J. Phys. Chem., $87: 2657-2664,1983$.

4 B. C. Garrett. Perspective on "The transition state method," Wigner E. (1938) Trans. Faraday Soc. 34:29-41. Theor. Chem. Acc., 103:200-204, 2000.

5 G. A. Petersson. Perspective on "The activated complex in chemical reactions," Eyring, H. (1995) J. Chem. Phys. 3: 107. Theor. Chem. Acc., 103:190-195, 2000.

6 E. Wigner. The transition state method. Trans. Faraday Soc., 34:29-41, 1938.

7 M. S. Child and E. Pollak. Analytical reaction dynamics: Origin and implications of trapped periodic trajectories. The Journal of Chemical Physics, 73:4365, 1980.

8 E. Pollak and M. S. Child. Classical mechanics of a collinear exchange reaction: A direct evaluation of the reaction probability and product distribution. The Journal of Chemical Physics, 73:4373, 1980 .

9 E. Pollak, M. S. Child, and P. Pechukas. Classical transition state theory: a lower bound to the reaction probability. The Journal of Chemical Physics, 72(3):1669-1678, 1980.

10 P. Pechukas and E. Pollak. Classical transition state theory is exact if the transition state is unique. The Journal of Chemical Physics, 71:2062, 1979.

11 E. Pollak and P. Pechukas. Transition states, trapped trajectories, and classical bound states embedded in the continuum. The Journal of Chemical Physics, 69:1218, 1978.

12 P. Pechukas and E. Pollak. Trapped trajectories at the boundary of reactivity bands in molecular collisions. The Journal of Chemical Physics, 67(12):5976-5977, 1977.

13 E. Pollak and P. Pechukas. Unified statistical model for" complex" and" direct" reaction mechanisms: A test on the collinear $\mathrm{H}+\mathrm{H}_{2}$ exchange reaction. The Journal of Chemical Physics, 70(1):325-333, 1979. 
14 R. Devogelare and M. Boudart. Contribution to the theory of reaction rates. J. Chem. Phys., 23:1236-1244, 1955.

15 S. Wiggins. On the geometry of transport in phase space I. Transport in $k$ degree-of-freedom Hamiltonian systems, $2 \leq k<\infty$. Physica D, 44:471-501, 1990.

16 R. E. Gillilan and G. S. Ezra. Transport and turnstiles in multidimensional Hamiltonian mappings for unimolecular fragmentation: Application to van der Waals predissociation. J. Chem. Phys., 94:2648-2668, 1991.

17 R.E. Gillilan and W.P Reinhardt. Barrier recrossing in surface diffusion: a phase space perspective. Chemical Physics Letters, 156:478-482, 1989.

18 R.E. Gillilan. Invariant surfaces and phase space flux in three-dimensional surface diffusion. The Journal of Chemical Physics, 93:5300, 1990.

19 S. Wiggins, L. Wiesenfeld, C. Jaffé, and T. Uzer. Impenetrable barriers in phase-space. Physical Review Letters, 86(24):5478-5481, 2001.

20 T. Uzer, C. Jaffé, J. Palacián, P. Yanguas, and S. Wiggins. The geometry of reaction dynamics. Nonlinearity, 15(4):957, 2002.

21 H. Waalkens, R. Schubert, and S. Wiggins. Wigner's dynamical transition state theory in phase space: classical and quantum. Nonlinearity, 21(1):R1, 2008.

22 C. B. Li, A. Shoujiguchi, M. Toda, and T. Komatsuzaki. Definability of no-return transition states in the high-energy regime above the reaction threshold. Physical review letters, $97(2): 28302,2006$.

23 A. Allahem and T. Bartsch. Chaotic dynamics in multidimensional transition states. submitted to Arxiv.org, 2012.

24 M. Inarrea, J. F. Palacian, A. I. Pascual, and J. P. Salas. Bifurcations of dividing surfaces in chemical reactions. J. Chem. Phys., 135:014110, 2011.

25 D. G. Yang. Breakdown of normal hyperbolicity for a family of invariant manifolds with generalized Lyapunov type numbers uniformly bounded below their critical values. submitted to Arxiv.org, 2009.

26 N. Fenichel. Persistence and smoothness of invariant manifolds for flows. Indiana Univ. Math. $J, 21(193-226): 1972,1971$.

27 N. Fenichel. Asymptotic stability with rate conditions. Indiana Univ. Math. J., 23:1109-1137, 1974. 
28 N. Fenichel. Asymptotic stability with rate conditions, ii. Indiana Univ. Math. J., 26:81-93, 1977.

29 M. W. Hirsch, C. Pugh, and M. Shub. Invariant manifolds. Bull. Amer. Math. Soc., 76(5):10151019, 1970.

30 S. Wiggins. Normally hyperbolic invariant manifolds in dynamical systems, volume 105. Springer, 1994.

31 A. Delshams, M. Gidea, and P. Roldan. Transition map and shadowing lemma for normally hyperbolic invariant manifolds. ArXiv e-prints, April 2012.

32 S. V. Bolotin and D. V. Treschev. Remarks on the definition of hyperbolic tori of hamiltonian systems. Regular and Chaotic dynamics, 5(4):401-412, 2000.

33 For our applications this means everywhere, except at possible isolated points in the phase space.

34 P. Collins, G. S. Ezra, and S. Wiggins. Isomerization dynamics of a buckled nanobeam. arXiv preprint arXiv:1206.3929, 2012.

35 E. Thiele. Comparison of the classical theories of unimolecular reactions. The Journal of Chemical Physics, 36(6):1466-1472, 1962.

36 E. Thiele. Comparison of the classical theories of unimolecular reactions. II. A model calculation. The Journal of Chemical Physics, 38(8):1959-1966, 1963.

37 G. S. Ezra, H. Waalkens, and S. Wiggins. Microcanonical rates, gap times, and phase space dividing surfaces. The Journal of Chemical Physics, 130(16):164118, 2009.

38 V. I. Arnol'd. Mathematical methods of classical mechanics, volume 60. Springer, 1989.

39 J. Binney, O. E. Gerhard, and P. Hut. Structure of surfaces of section. Monthly Notices of the Royal Astronomical Society, 215:59, 1985.

40 H-D. Meyer. Theory of the Liapunov exponents of Hamiltonian systems and a numerical study on the transition from regular to irregular classical motion. The Journal of Chemical Physics, 84(6):3147-3161, 1986.

41 M. Toller, G. Jacucci, G. DeLorenzi, and C. P. Flynn. Theory of classical diffusion jumps in solids. Physical Review B, 32(4):2082, 1985.

42 R. S. MacKay. Flux over a saddle. Physics Letters A, 145(8):425-427, 1990.

43 H. Waalkens and S. Wiggins. Direct construction of a dividing surface of minimal flux for multi-degree-of-freedom systems that cannot be recrossed. Journal of Physics A: Mathematical 
and General, 37(35):L435, 2004.

44 P. Brumer, D. E. Fitz, and D. Wardlaw. Time delay for bimolecular collisions: Utility of the spectral theorem in the classical limit. The Journal of Chemical Physics, 72:386, 1980.

45 E. Pollak. A classical spectral theorem in bimolecular collisions. The Journal of Chemical Physics, 74:6763, 1981.

46 H. Waalkens, A. Burbanks, and S. Wiggins. Efficient procedure to compute the microcanonical volume of initial conditions that lead to escape trajectories from a multidimensional potential well. Physical review letters, 95(8):84301, 2005.

47 H. Waalkens, A. Burbanks, and S. Wiggins. A formula to compute the microcanonical volume of reactive initial conditions in transition state theory. Journal of Physics A: Mathematical and General, 38(45):L759, 2005.

48 N. B. Slater. New formulation of gaseous unimolecular dissociation rates. The Journal of Chemical Physics, 24(6):1256-1257, 1956.

49 N. B. Slater. Theory of unimolecular reactions. Cornell University Press Ithaca, New York, 1959.

50 R. S. Dumont and P. Brumer. Dynamical theory of statistical unimolecular decay. The Journal of Physical Chemistry, 90(16):3509-3516, 1986.

51 B. K. Carpenter. Nonexponential decay of reactive intermediates: new challenges for spectroscopic observation, kinetic modeling and mechanistic interpretation. Journal of physical organic chemistry, 16(11):858-868, 2003.

52 D. L. Bunker. Monte Carlo Calculation of Triatomic Dissociation Rates. I. NO and O. The Journal of Chemical Physics, 37:393, 1962.

53 D. L. Bunker. Monte Carlo calculations. IV. Further studies of unimolecular dissociation. The Journal of Chemical Physics, 40:1946, 1964.

54 D. L. Bunker and W. L. Hase. On non-RRKM unimolecular kinetics: Molecules in general, and CHNC in particular. The Journal of Chemical Physics, 59:4621, 1973.

55 D. L. Bunker. Theory of Elementary Gas Reaction Rates. Pergamon Press, 1966.

56 D. L. Bunker and M. Pattengill. Monte Carlo Calculations. VI. A Re-evaluation of the RRKM Theory of Unimolecular Reaction Rates. The Journal of Chemical Physics, 48(2):772-776, 1968.

57 W. L. Hase. Dynamics of Unimolecular Reactions. In W. H. Miller, editor, Modern Theoretical Chemistry, volume 2, pages 121-170. Plenum, New York, 1976. 
58 S. Y. Grebenshchikov, R. Schinke, and W. L. Hase. State-specific dynamics of unimolecular dissociation. Comprehensive Chemical Kinetics, 39:105-242, 2003.

59 U. Lourderaj and W.L. Hase. Theoretical and computational studies of non-rrkm unimolecular dynamics. J. Phys. Chem. A, 113(11):2236-2253, 2009.

60 H. Hancock. Elliptic integrals. John Wiley \& Sons, inc., 1917. 


\section{Figure captions}

FIG. 1: Phase portrait for example 1 (cf. sec. III A 1). The phase space is the Cartesian product (denoted by "X") of the phase spaces for the uncoupled subsystems.

FIG. 2: Phase portrait for example 2 (cf. sec. III A 2). The phase space is the Cartesian product (denoted by "X") of the phase spaces for the uncoupled subsystems.

FIG. 3: Dividing surface for example 1 (eq. (3.9) for three different energies. Parameter $\alpha_{1}=0.8$. (a) $E=0.5$. (b) $E=\alpha_{1}^{2}$. (c) $E=1.0$.

FIG. 4: Flux $\phi(E)$ across the DS for example 1 as a function of energy $E\left(\alpha_{1}=0.8\right)$. FIG. 5: Dividing surface for example 2 (eq. (3.9) ) for three different energies. Parameters $\alpha_{1}=\alpha_{2}=1$. (a) $E=-0.15$. (b) $E=0$. (c) $E=1.0$.

FIG. 6: Flux $\phi(E)$ across the DS for example 2 as a function of energy $E\left(\alpha_{1}=\alpha_{2}=1\right)$. FIG. 7: Gap time distributions. (a) Example 1, energy $E_{T}=5$. (b) Example 2, energy $E_{T}=5$. Note the existence of a singularity in the gap time distributions for both cases. 

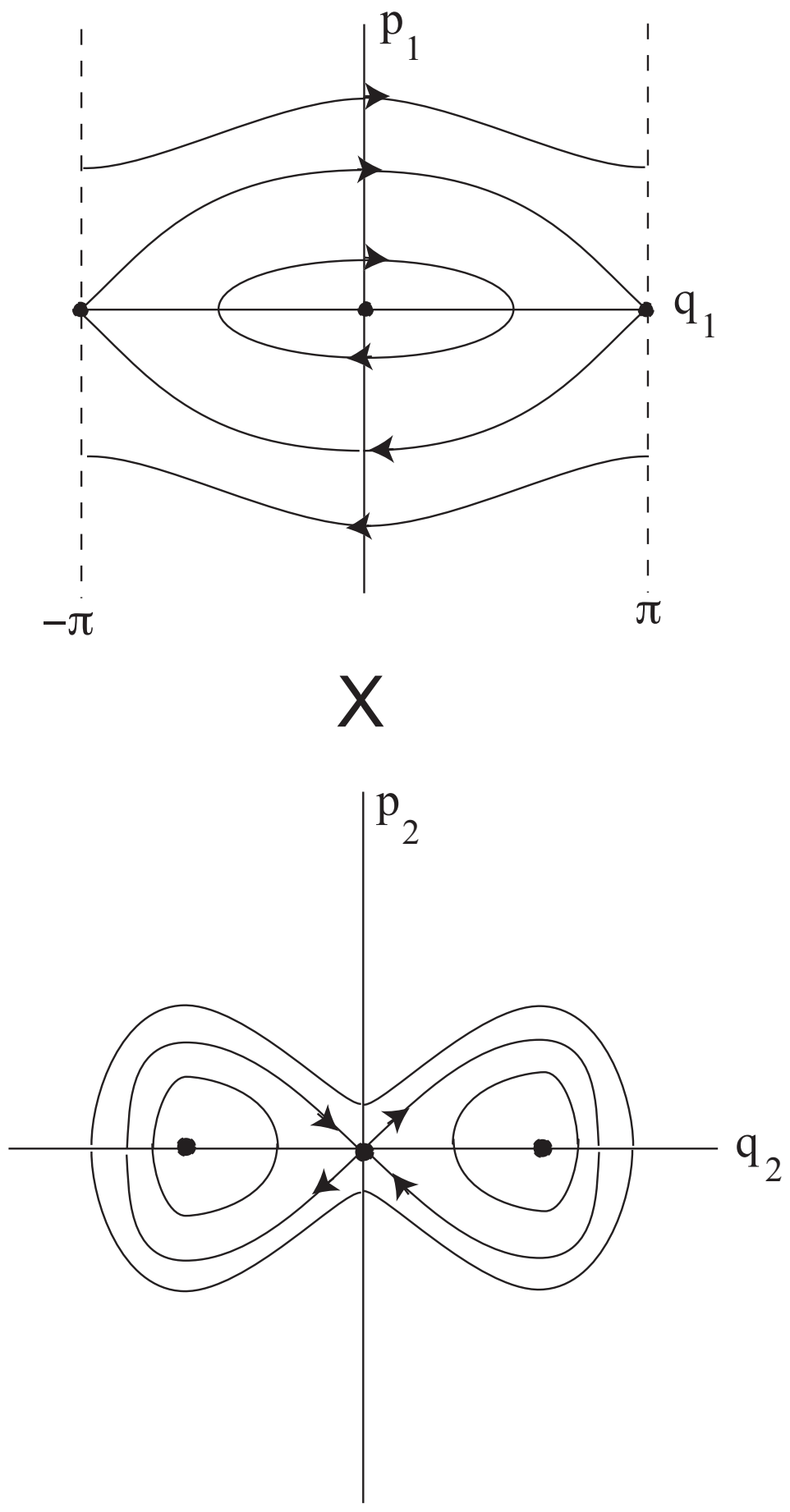

FIGURE 1 

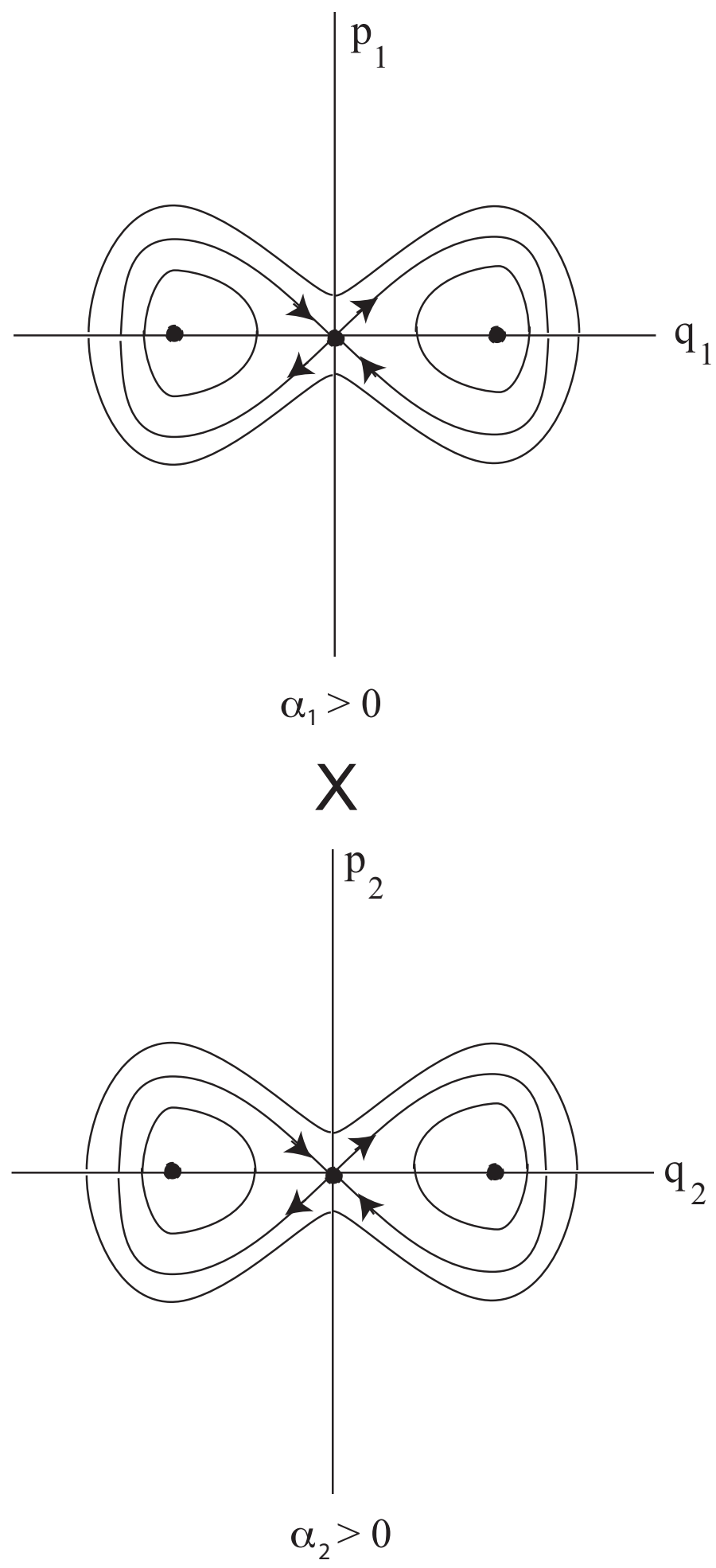

FIGURE 2 

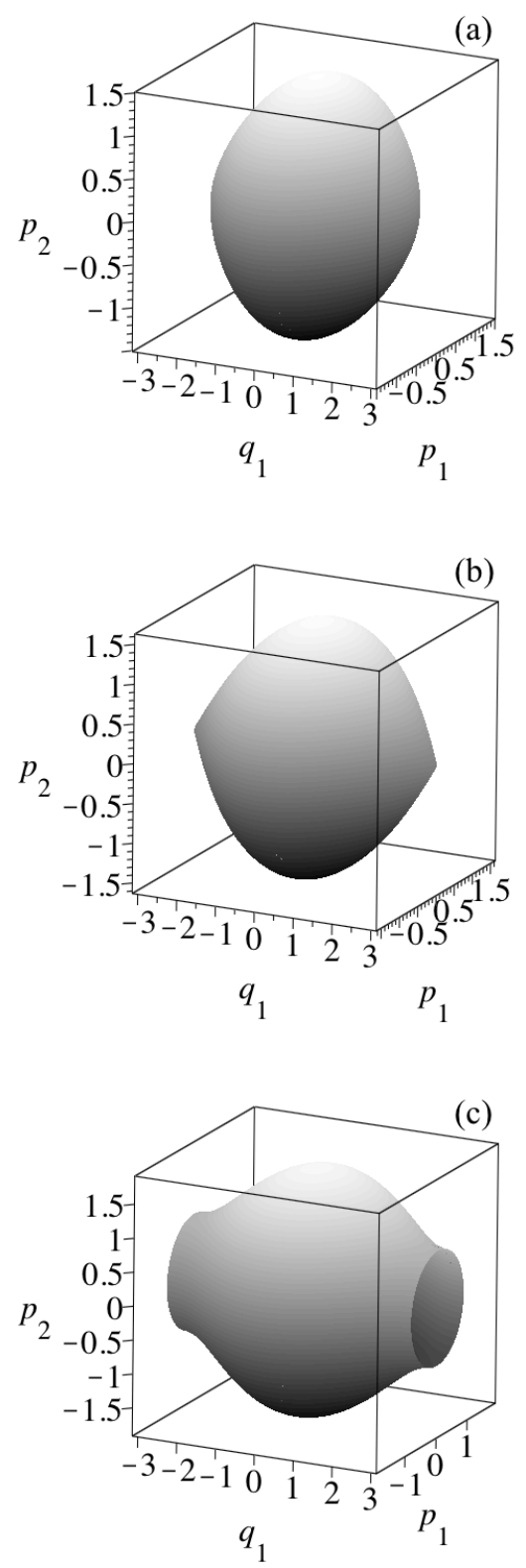

FIGURE 3 


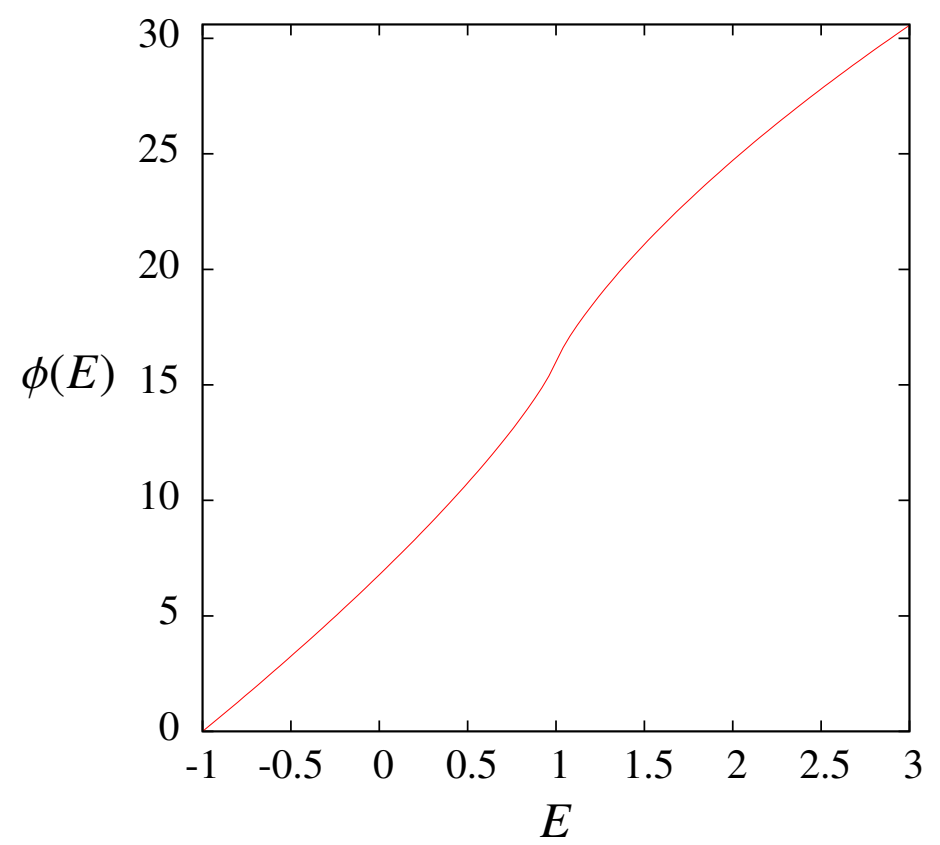

FIGURE 4 

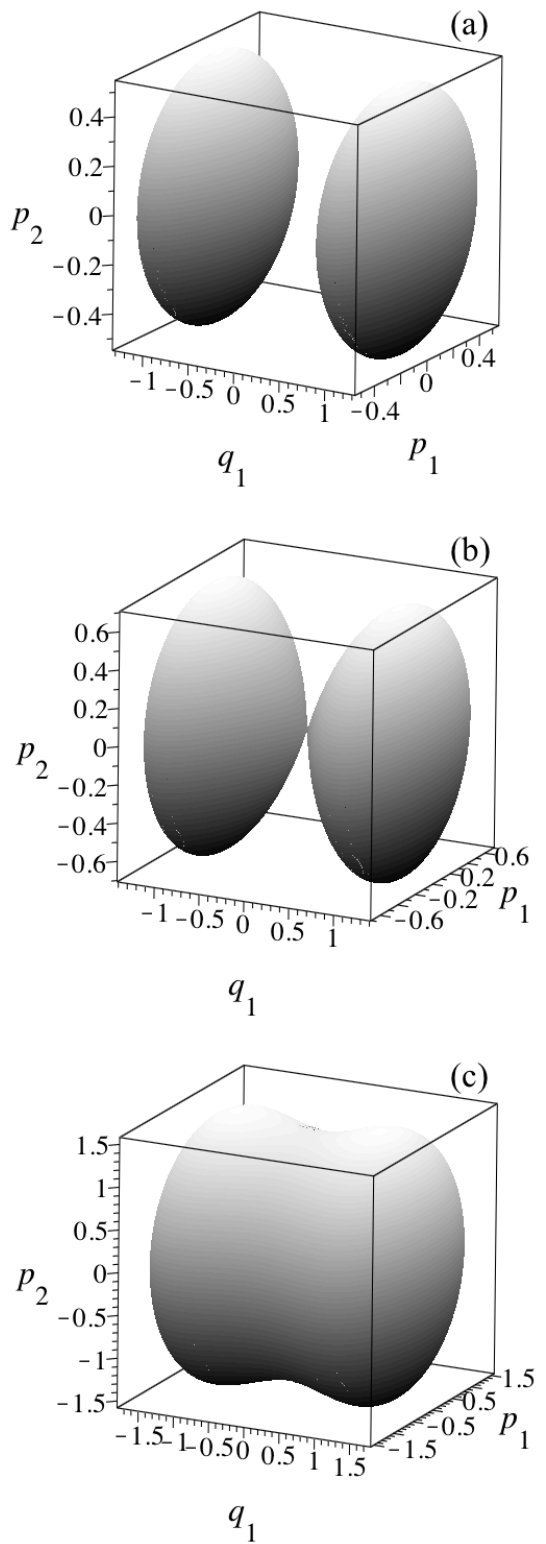

FIGURE 5 


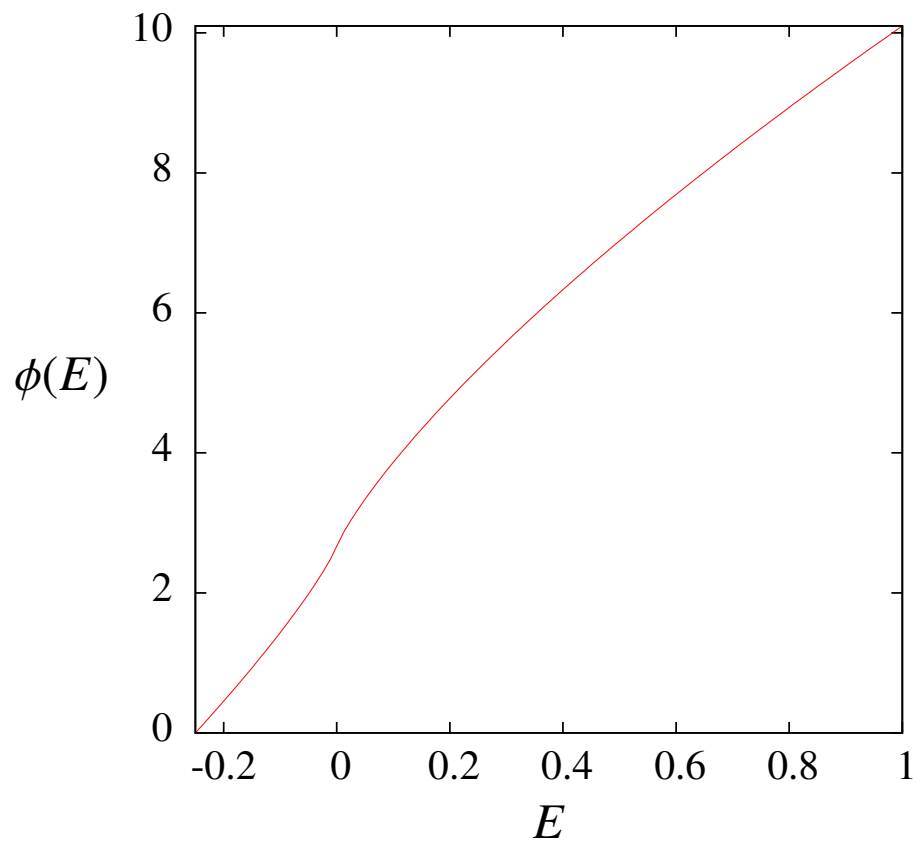

FIGURE 6 

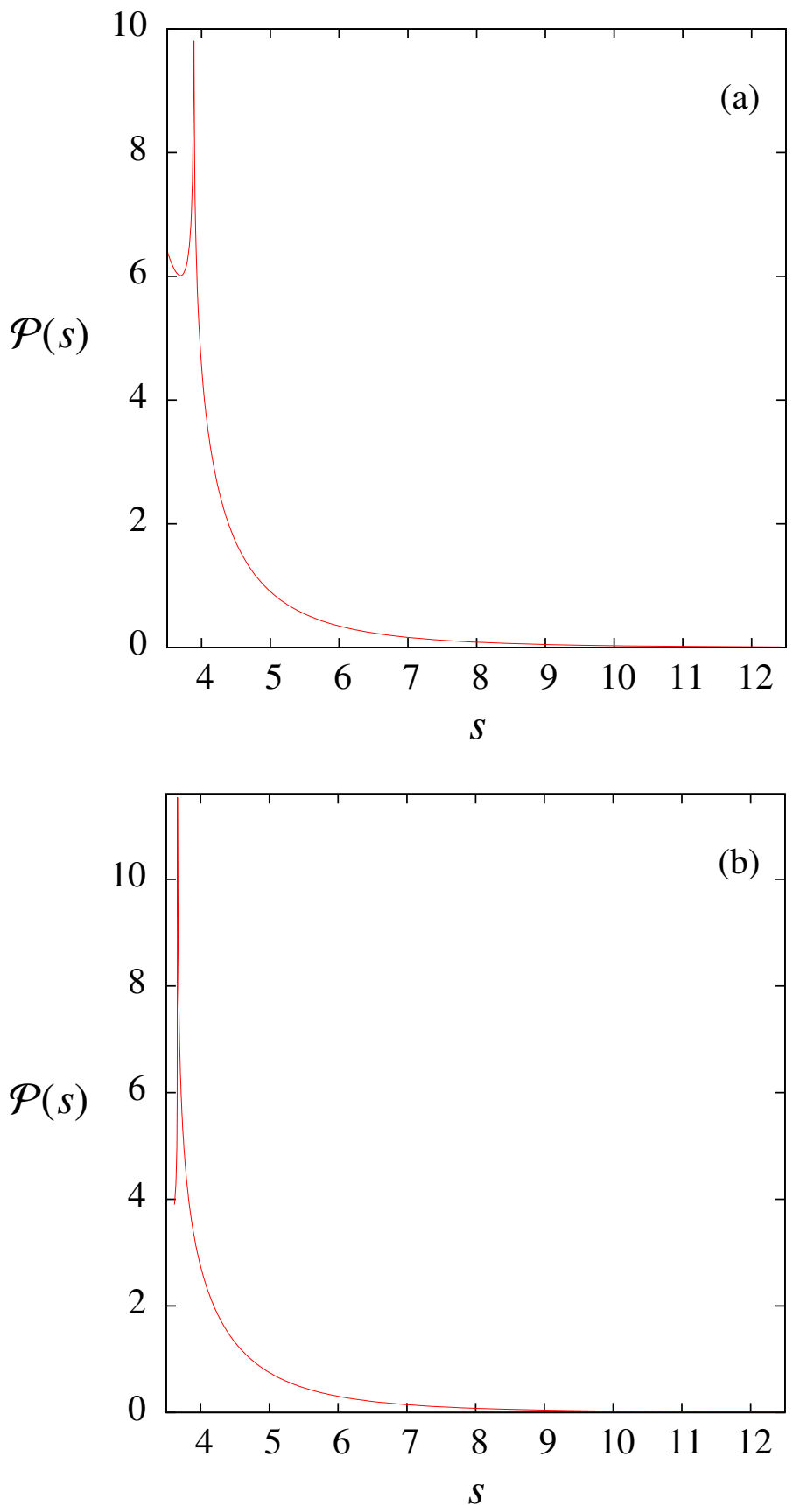

\section{FIGURE 7}

